\title{
Complement to thermodynamics of dyonic Taub-NUT-AdS spacetime
}

\author{
Robert B. Mann, ${ }^{a}$ Leopoldo A. Pando Zayas ${ }^{b, c}$ and Miok Park ${ }^{d}$ \\ ${ }^{a}$ Department of Physics and Astronomy, University of Waterloo, \\ Waterloo, Ontario, N2L 3G1, Canada \\ ${ }^{b}$ Leinweber Center for Theoretical Physics, University of Michigan, \\ Ann Arbor, $M I$ 48109, U.S.A. \\ ${ }^{c}$ The Abdus Salam International Centre for Theoretical Physics, \\ 34014 Trieste, Italy \\ ${ }^{d}$ School of Physics, Korea Institute for Advanced Study, \\ Seoul 02455, Republic of Korea \\ E-mail: rbmann@uwaterloo.ca, lpandoz@umich.edu, miokpark@kias.re.kr
}

ABstract: We examine the thermodynamics of Euclidean dyonic Taub-Nut/Bolt-AdS 4 black holes for a variety of horizon geometries to understand how gauge field regularity conditions influence the thermodynamic relations. We find several distinct features that distinguish the NUT-charged case from its dyonic Reissner-Nordstrom counterpart. For the Nut solution, the gauge field vanishes at the horizon and so regularity is ensured. For the Bolt solution we find that the norm of the gauge field is required to vanish at the horizon in order to satisfy both regularity and the first law of thermodynamics. This regularity condition yields a constraint on the electric and magnetic charges and so reduces cohomogeneity of the system; for spherical horizons, the regularity condition removing the Misner string singularity further reduces cohomogeneity. We observe that bolt solutions with increasing electric charge have positive heat capacity, but upon turning on the magnetic charge to make the solution dyonic, we find that the properties of the uncharged one are retained, having both positive and negative heat capacity. We also study the extremal Bolt solution, finding that Misner string disappears at the horizon in the zero temperature limit. We find that the extremal solution has finite-temperature-like behaviour, with the electric potential playing a role similar to temperature.

KEYwords: Black Holes, Classical Theories of Gravity

ArXiv EPrint: 2012.13506 


\section{Contents}

1 Introduction 1

2 Recap of thermodynamics of Uncharged TN/TB-AdS 4

2.1 Uncharged TN-AdS 5

2.2 Uncharged TB-AdS 6

3 Thermodynamics of dyonic TN/TB-AdS 9

$\begin{array}{ll}3.1 \text { Dyonic TN-AdS solution and thermodynamics } & 10\end{array}$

$\begin{array}{lll}3.2 & \text { Dyonic TB-AdS } & 12\end{array}$

$\begin{array}{lll}3.2 .1 & \text { Thermodynamics for } \kappa \neq 1 & 13\end{array}$

$\begin{array}{ll}3.2 .2 \text { Thermodynamics for } \kappa=1 & 14\end{array}$

4 Zero temperature limit of dyonic TB-AdS $\quad 19$

$\begin{array}{llr}5 & \text { Conclusion } & 21\end{array}$

A Thermodynamics of the Dyonic RN black hole 23

B Electric potential for dyonic Taub-NUT-AdS 24

$\begin{array}{ll}\text { C Solution for } r_{b} \text { in terms of other parameters } & 25\end{array}$

$\begin{array}{ll}\text { D Gauge field strength } & 25\end{array}$

\section{Introduction}

One of the more interesting solutions to the Einstein equations is Taub-NUT spacetime, a generalization of the Schwarzschild spacetime originally obtained by Taub in 1951 [1] and later rediscovered by Newman, Tamburino, and Unti in 1963 [2]. This spacetime is asymptotically locally flat: its Riemann tensor approaches zero at large distance, but it is only locally isomorphic to Minkowski spacetime in this regime. Its (anti) de Sitter ((A)dS) version, Taub-NUT-(A)dS spacetime, is likewise asymptotically locally (A)dS: its Riemann tensor

$$
R_{\alpha \beta \mu \nu} \rightarrow \mp \frac{1}{l^{2}}\left(g_{\alpha \mu} g_{\beta \nu}-g_{\alpha \nu} g_{\beta \mu}\right)
$$

at large distance where $l$ is the (A) $\mathrm{dS}_{4}$ length. These Taub-NUT(-(A)dS) spacetimes also have a mass parameter $(m)$ and a NUT charge $(s)$, with horizons at $g_{t t}\left(r_{+}\right)=0$. However the Kretschmann scalar does not diverge at the origin $r=0$ unless $s=0$. Its extension to the charged case is firstly studied in [3] for an asymptotically locally flat spacetime and in $[4,5]$ for an asymptotically locally AdS spacetime. 
Interestingly, these spacetimes have peculiar properties when their horizon geometry is a sphere due to the non-trivial fibration of time on $S^{2}$, which induces a spurious singularity in a given coordinate system. Known as a Misner string [6], this singularity is analogous to the Dirac string of electromagnetism, and the NUT charge is a gravitomagnetic charge analogous to a magnetic monopole. Hence any Taub-NUT solution with nonzero $m$ and $s$ is a gravitational dyon. Like the Dirac string, the Misner string that appears along the $\theta=\pi$ (or $\theta=0$ ) axis can be eliminated by introducing an additional time coordinate in a neighbourhood $\theta=\pi$ (or $\theta=0$ ) that is regular there, and then gluing this region to its complement; a similar construction can be made for a Misner string along any axis. However this regularity condition automatically requires the time coordinate to be periodic $t \sim t+8 \pi s$, which yields closed timelike curves (CTCs) through every point. While this is a benign situation in Euclidean space, it violates causality in Lorentzian spacetime, and so the latter has generally been thought to be pathological.

Since the Taub-NUT-((A)dS) spacetime retains these peculiar features, understanding their thermodynamic properties were challenging, mainly due to the violation of Bekenstein-Hawking formula, but successfully studied in [7-9]. Moreover, as this solution can be inherited from M-theory or supergravity theory, its higher dimensional solutions are studied in $[10,11]$ and its supersymmetric properties are found in [12]. Also the asymptotically locally AdS spacetime can be considered as a natural extension of the AdS/CFT correspondence and holographic matching with its boundary field theory was studied in $[13,14]$.

Recently a new perspective on these spacetimes has emerged, based on studies [15] showing that the Misner string in Lorentzian Taub-NUT spacetime is transparent for geodesics, implying that the spacetime becomes geodesically complete. Thus imposing time periodicity is not necessary and no causality problem occurs. Based on this argument, thermodynamic properties of Lorentzian Taub-NUT-AdS spacetime were subsequently studied [16-18], with the perspective that the first law has full cohomogeneity by treating the NUT charge as an independent thermodynamic variable, a Misner charge whose conjugate variable is called the Misner potential, with these new terms included in the first law and free energy.

Here we consider Euclidean dyonic Taub-NUT-AdS 4 spacetime and investigate its thermodynamic properties for all horizon geometries characterized by a parameter $\kappa$, where $\kappa=1$ corresponds to spherical geometries, $\kappa=0$ to locally flat geometries, and $\kappa<0$ to hyperbolic geometries. We also consider the role played by the Misner string if $\kappa=1$. This extends previous studies [19-22]; our aim here is to understand how regularity conditions of the gauge field affect the thermodynamic relations. For the dyonic RN black hole, the first law of thermodynamics is not strongly affected by the regularity condition of the gauge field, and an electric and magnetic charge are independently present in the thermodynamic relations as illustrated in appendix A. However for the dyonic Taub-NUT-AdS solution, we find that the regularity condition, which relates the electric and magnetic charges, is essential for satisfying the first law of thermodynamics.

To start with, we perform the Wick-rotation $t \rightarrow-i \tau$ and $s \rightarrow-i \chi$ to Euclidean space from the Lorentzian solution. The U(1) isometry group associated with the Killing vector 
$\xi=\partial_{\tau}$ then has either a zero-dimensional fixed point set (the so-called nut solution, or TN-AdS) or a two-dimensional fixed point set (the so-called bolt solution, or TB-AdS). When the horizon geometry is that of a sphere $(\kappa=1)$ it is of special interest because of the existence of the Misner string, which is removed by imposing the time periodicity as stated above. The Hawking temperature is then constrained to be $\frac{1}{8 \pi \chi}$, and the Misner string contributes to the entropy. Thus the entropy is not just the area of the fixed point set of $\xi$, which is the Bekenstein-Hawking entropy formula $[8,9]$. With these considerations, the thermodynamic properties of (a charged) Taub-NUT-AdS spacetime for $\kappa=1$ have been studied [19-21]; for the uncharged TB-AdS case there is a maximum value of the NUT charge. Each value of NUT charge has two branches [19], characterized by increasing/decreasing horizon radius as the NUT charge increases to its maximum value. We shall refer to them as small and large TB-AdS solutions. They respectively have negative and positive heat capacities.

The TN-AdS solution demands that $P=i Q$, which in turn implies the norm of the gauge field vanishes at the horizon. However for TB-AdS, the zero value of this norm must be imposed (rather than having a finite value) to ensure the validity of the thermodynamic relations. One way of satisfying this regularity condition for TB-AdS is to relate electric and magnetic charges:

$$
P=\frac{2 i Q \chi r_{+}}{r_{+}^{2}+\chi^{2}}
$$

implying $Q$ and $P$ are no longer independent. This reduces the cohomogeneity of the system. Removal of the Misner string if $\kappa=1$ yields a condition that further reduces the cohomogeneity in the thermodynamic relations. In this case the following thermodynamic relations

$$
d E=T d S+\Phi_{E} d Q, \quad F=E-T S-\Phi_{E} Q
$$

are satisfied.

We also find that these thermodynamic arguments are valid in the zero temperature limit and study the near horizon geometry, which is $A d S_{2} \times \mathcal{M}_{\kappa}$. For $\kappa=1$, it is possible to make sense of the thermodynamics provided the temperature is not identified via Misner string periodicity arguments. We observe that, upon replacing the temperature with the electric potential [23], the extremal TB-AdS solution exhibits similar thermodynamic behaviour to that of the uncharged TB-AdS solution.

Our manuscript is organized as follows. In section 2, we review the thermodynamics for the uncharged TN/TB-AdS. We shall work at fixed AdS length, not working in the extended thermodynamic phase space [24]. We check the first law of black hole thermodynamics and free energy and plotted the horizon of TB-AdS, entropy and heat capacity as functions of the NUT charge $\chi$, which by regularity requirements is the inverse of temperature for $\kappa=1$. In section 3, we revisit the thermodynamics of the charged TN-AdS and TB-AdS. We recover some results obtained previously [21], but go beyond this by considering all values of $\kappa$, calculating the heat capacity and other relevant thermodynamic quantities before imposing the regularity condition. We find that $P$ and $Q$ are related by metric requirements for TN-AdS and by the regularity condition of the gauge field at 
the horizon of TB-AdS. We impose the regularity condition after obtaining the various thermodynamic quantities to show that applying the regularity condition is necessary for the thermodynamic relations to hold. In section 4, we then investigate the near horizon geometry in the zero temperature limit, and study the resultant thermodynamic relations. Then we summarize our results in section 5 .

\section{Recap of thermodynamics of Uncharged TN/TB-AdS}

The Einstein-Hilbert action with cosmological constant and Gibbons-Hawking term in four dimensional spacetime is

$$
\begin{aligned}
I= & \frac{1}{16 \pi G_{4}} \int_{\mathcal{M}} d^{4} x \sqrt{-g}\left(R+\frac{6}{l^{2}}\right) \\
& +\frac{1}{8 \pi G_{4}} \int_{\partial \mathcal{M}} d^{3} x \sqrt{-h} K-\frac{1}{8 \pi G_{4}} \int_{\partial \mathcal{M}} d^{3} x \sqrt{-h}\left(\frac{2}{l}+\frac{l}{2} R_{3}(h)\right)
\end{aligned}
$$

where $l$ is the radius of $A d S_{4}$ spacetime, $K$ is the trace of extrinsic curvature on the boundary at spatial infinity, and the last term is the boundary counterterm action $I_{c t}[9$, $25,26]$.

The variation of the action with respect to the metric yields the equations of motion

$$
R_{\mu \nu}-\frac{1}{2} g_{\mu \nu} R-\frac{3}{l^{2}} g_{\mu \nu}=0
$$

with the solution

$$
d s^{2}=-f(r)(d t+2 s \lambda(\theta) d \phi)^{2}+\frac{d r^{2}}{f(r)}+\left(r^{2}+s^{2}\right)\left(d \theta^{2}+Y(\theta)^{2} d \phi^{2}\right)
$$

where $s$ is the NUT charge,

$$
f(r)=\frac{l^{-2}\left(r^{2}+s^{2}\right)^{2}+\left(\kappa+4 l^{-2} s^{2}\right)\left(r^{2}-s^{2}\right)-2 M r}{r^{2}+s^{2}}
$$

and

$$
\lambda(\theta)=\left\{\begin{array}{ll}
\cos \theta \\
-\theta \\
-\cosh \theta
\end{array} \quad, \quad Y(\theta)= \begin{cases}\sin \theta & \text { for } \kappa=1 \\
1 & \text { for } \kappa=0 \\
\sinh \theta & \text { for } \kappa=-1\end{cases}\right.
$$

characterize the three possible geometries of the constant-curvature horizon, located at the largest root of $f(r)=0$.

To explore the thermodynamic properties of the spacetime (2.3), we shall compute its free-energy under various circumstances. Performing the Wick-rotation $t \rightarrow-i \tau$ and $s \rightarrow-i \chi$, the Euclidean action and associated metric are

$$
\begin{aligned}
I_{E} & =-\frac{1}{16 \pi G_{4}} \int d^{4} x \sqrt{g}\left(R+\frac{6}{l^{2}}\right)-\frac{1}{8 \pi G_{4}} \int d^{3} x \sqrt{h}\left(K-\frac{2}{l}-\frac{l}{2} R_{3}\right) \\
d s_{\mathrm{E}}^{2} & =f_{E}(r)(d \tau+2 \chi \lambda(\theta) d \phi)^{2}+\frac{d r^{2}}{f_{E}(r)}+\left(r^{2}-\chi^{2}\right)\left(d \theta^{2}+Y(\theta)^{2} d \phi^{2}\right), \\
f_{E} & =\frac{l^{-2}\left(r^{2}-\chi^{2}\right)^{2}+\left(\kappa-4 l^{-2} \chi^{2}\right)\left(r^{2}+\chi^{2}\right)-2 M r}{r^{2}-\chi^{2}}
\end{aligned}
$$


This solution has an horizon at $r=r_{+}$from $f_{E}\left(r_{+}\right)=0$. There are two qualitatively distinct solutions that are characterized by the fixed point sets associated with the Killing vector field $\xi=\partial_{\tau}$. If the radius is the same as the NUT charge $\left(r_{+}=\chi\right)$, the fixed point set is zero-dimensional, and is referred to as the Taub-Nut-AdS (TN-AdS) solution. Otherwise $r_{+}>\chi$, in which case the fixed point sets is two-dimensional and is called the Taub-Bolt-AdS(TB-AdS) solution.

\subsection{Uncharged TN-AdS}

The TN-AdS solution is obtained when $f_{\mathrm{E}}(r)$ has a root at $r=r_{+}=\chi$. The Euclidean metric function then can be written as

$$
f_{n}(r)=\frac{(r-\chi)\left(\kappa l^{2}+r^{2}+2 r \chi-3 \chi^{2}\right)}{l^{2}(r+\chi)}
$$

where in $(2.8)$

$$
M=M_{n}=\chi \kappa-\frac{4 \chi^{3}}{l^{2}}
$$

since $r_{+}=\chi$. The Hawking temperature is easily calculated as

$$
T_{n}=\frac{1}{4 \pi} f_{n}^{\prime}(\chi)=\frac{\kappa}{8 \pi \chi}
$$

and this automatically agrees with the condition for removal of the Misner string. When $\kappa=-1, f_{n}$ becomes negative near the horizon limit $r=\chi+\epsilon$. This happens because $r=\chi$ is not the outermost horizon, but instead is contained in a bolt solution whose horizon is $\sqrt{4 \chi^{2}-\kappa l^{2}}-\chi[27]$. Thus there is no hyperbolic TN-AdS solution. We therefore only consider the $\kappa=0$ and $\kappa=1$ cases for TN-AdS, noting that the former is a zerotemperature extremal case.

The extrinsic curvature and the three dimensional Ricci scalar are calculated as

$$
\begin{aligned}
K & =\frac{(r-\chi)\left(3 r^{2}+9 r \chi+4 \chi^{2}\right)+l^{2}(2 r+\chi) \kappa}{l^{2}(r+\chi)^{2} \sqrt{f_{n}(r)}}, \\
R_{3} & =-\frac{2 \chi^{2}\left(\kappa l^{2}+r^{2}+2 r \chi-3 \chi^{2}\right) \lambda^{\prime}(\theta)^{2}+2 l^{2}(r+\chi)^{2} Y(\theta) Y^{\prime \prime}(\theta)}{l^{2}(r-\chi)(r+\chi)^{3} Y(\theta)^{2}} .
\end{aligned}
$$

From these we obtain

$$
\begin{aligned}
I_{\mathrm{E}} & =\frac{\beta \omega}{8 \pi G}\left[3 M_{n}-2 \kappa R-\frac{2\left(R^{3}-4 R \chi^{2}-\chi^{3}\right)}{l^{2}}+\mathcal{O}\left(\frac{1}{R}\right)\right]+I_{c t}, \\
I_{\mathrm{ct}} & =\frac{\beta \omega}{8 \pi G l} \sqrt{f_{n}(R)}\left[2\left(R^{2}-\chi^{2}\right)+\kappa l^{2}-\frac{l^{2} \chi^{2} f_{n}(R)}{\left(R^{2}-\chi^{2}\right)}\right], \\
I_{\text {ren. }} & =\lim _{R \rightarrow \infty} I_{\mathrm{E}}=\frac{\beta \omega}{8 \pi G}\left(M_{n}+\frac{2 \chi^{3}}{l^{2}}\right)=\frac{\beta \omega \chi}{8 \pi G}\left(\kappa-\frac{2 \chi^{2}}{l^{2}}\right)
\end{aligned}
$$

for the on-shell Euclidean action (2.6), where $\omega=\int_{0}^{2 \pi} d \phi \int_{\theta_{\min }(\phi)}^{\theta_{\max }(\phi)} d \theta Y(\theta)$ is the volume element of the transverse space, ${ }^{1}$ and $\beta$ is the imaginary time periodicity, which becomes

\footnotetext{
${ }^{1}$ For $\kappa=1, \theta_{\max }(\phi)=\pi$ and $\theta_{\min }(\phi)=0$; for other values of $\kappa$ these quantities depend on the identifications made in the transverse space [28].
} 
the inverse of temperature. For non-compact transverse spaces, we shall rescale our thermodynamic quantities by $\omega$.

The free energy is then given by definition

$$
F=-\frac{1}{\beta} \log Z \sim \frac{I_{\text {ren. }}}{\beta}
$$

where $Z$ is a partition function and we used a saddle point approximation in the second expression. The thermodynamic entropy and energy are obtained from

$$
S=\left(\beta \frac{\partial}{\partial \beta}-1\right) I_{\text {ren. }}, \quad E=\partial_{\beta} I_{\text {ren. }}
$$

yielding

$$
S=\frac{\omega \chi^{2}}{G}\left(\kappa-\frac{6 \chi^{2}}{l^{2}}\right), \quad E=\frac{\chi \omega}{4 \pi G}\left(\kappa-\frac{4 \chi^{2}}{l^{2}}\right)=\frac{\omega}{4 \pi G} M_{n} .
$$

These quantities satisfy the first law and free energy

$$
d E=T_{n} d S, \quad F=E-T_{n} S
$$

which is only valid for $\kappa=1$ case since there is no hyperbolic TN-AdS. For $\kappa=0$, the temperature is zero, we cannot determine the entropy using (2.18). However since the Misner string is not present, we expect that the entropy satisfies the Bekenstein-Hawking entropy and so becomes the area of the TN-AdS, which is zero.

\subsection{Uncharged TB-AdS}

For TB-AdS, the horizon is at $r=r_{b} \neq \chi$ and the mass parameter is

$$
M_{b}=\frac{-6 \chi^{2} r_{b}^{2}+r_{b}^{4}-3 \chi^{4}+\kappa\left(r_{b}^{2}+\chi^{2}\right) l^{2}}{2 l^{2} r_{b}}
$$

from (2.8). The extrinsic curvature and the three dimensional Ricci scalar are

$$
\begin{aligned}
K & =\frac{\left(-3 r^{2}+\chi^{2}\right) M_{b}+2 \kappa r^{3}+\left(3 r^{5}-14 r^{3} \chi^{2}+3 r \chi^{4}\right) l^{-2}}{\left(r^{2}-\chi^{2}\right)^{2} \sqrt{f_{\mathrm{E}}(r)}} \\
R_{3} & =\frac{-2 \chi^{2}\left(-2 M_{b} r+\kappa\left(r^{2}+\chi^{2}\right)+\left(r^{4}-6 r^{2} \chi^{2}-3 \chi^{4}\right) l^{-2}\right) \lambda^{\prime}(\theta)^{2}}{\left(r^{2}-\chi^{2}\right)^{3} Y(\theta)^{2}}-\frac{2 Y^{\prime \prime}(\theta)}{\left(r^{2}-\chi^{2}\right) Y(\theta)}
\end{aligned}
$$

yielding

$$
\begin{aligned}
I_{\mathrm{E}} & =\frac{\omega \beta}{8 \pi G}\left[3 M_{b}-\frac{\left(2 R^{3}+r_{b}^{3}-3 r_{b} \chi^{2}\right)}{l^{2}}+\left(\frac{8 \chi^{2}}{l^{2}}-2 \kappa\right) R+\mathcal{O}\left(\frac{1}{R}\right)\right]+I_{c t} \\
I_{\mathrm{ct}} & =\frac{\beta \omega}{8 \pi G l} \sqrt{f_{\mathrm{E}}(R)}\left[2\left(R^{2}-\chi^{2}\right)+\kappa l^{2}-\frac{l^{2} \chi^{2} f_{\mathrm{E}}(R)}{\left(R^{2}-\chi^{2}\right)}\right], \\
I_{\text {ren. }} & =\lim _{R \rightarrow \infty} I_{\mathrm{E}}=\frac{\omega \beta}{8 \pi G}\left[M_{b}-\frac{r_{b}^{3}-3 r_{b} \chi^{2}}{l^{2}}\right],
\end{aligned}
$$

for the Euclidean action. 


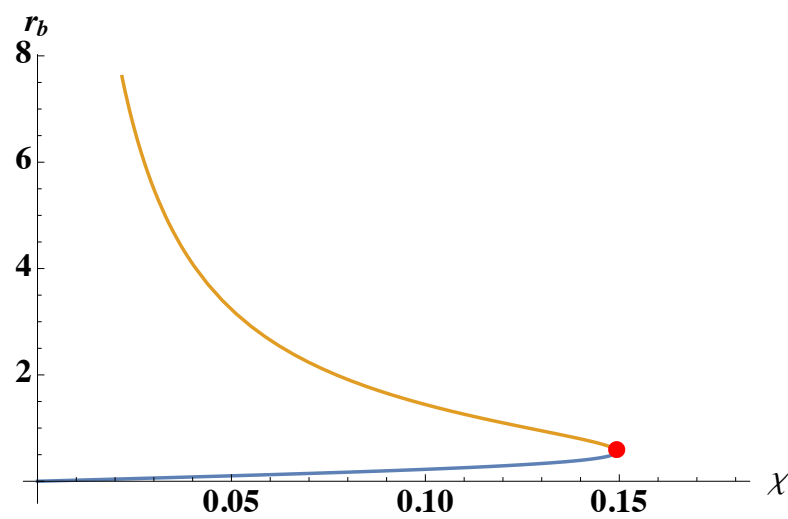

Figure 1. The horizon radius $r_{b}(2.29)$ vs. NUT charge for uncharged TB-AdS for $\kappa=1$ and $G=l=1$.

The Hawking temperature is

$$
T=\frac{1}{4 \pi} f_{\mathrm{E}}^{\prime}\left(r_{b}\right)=\frac{M_{b}\left(r_{b}^{2}+\chi^{2}\right)+\left(9 \chi^{4} r_{b}-2 \chi^{2} r_{b}^{3}+r_{b}^{5}\right) l^{-2}-2 \kappa r_{b} \chi^{2}}{2 \pi\left(\chi^{2}-r_{b}^{2}\right)^{2}}
$$

where for $\kappa=1$ we must also have

$$
T=\frac{1}{4 \pi} f_{\mathrm{E}}^{\prime}(\chi)=\frac{1}{8 \pi \chi}
$$

to remove the Misner string singularity. This constrains the thermodynamic phase space. Inserting (2.21) into (2.27) and equating the result to (2.28), the horizon radius is

$$
r_{b}=\frac{1}{12 \chi}\left(l^{2} \pm \sqrt{l^{4}+144 \chi^{4}-48 l^{2} \chi^{2}}\right)
$$

which we depict in figure 1 in units with $G=1$ and $l=1$. The blue/orange solid lines correspond to the negative/positive roots in (2.29). There is a maximum value of the NUT charge at

$$
\chi=\chi_{\max }=\frac{l}{2} \sqrt{\frac{1}{3}(2-\sqrt{3})}
$$

which we denote as a red dot in figure 1. Thus the NUT charge lies in the range $0 \leq$ $\chi \leq \chi_{\max }$ and has two branches that show increasing (the lower blue curve) and decreasing (the upper orange curve) behaviour of the radius as the NUT charge increases. These two branches have different characteristics in large $l$ limit as follows

$$
r_{b-} \sim 2 \chi, \quad r_{b+} \sim \frac{l^{2}}{6 \chi}
$$

The lower branch is smoothly connected TB in flat spacetime when $l$ is taken to infinity, whereas the upper branch does not smoothly go to TB in flat spacetime since $r_{b}$ diverges in the $l \rightarrow \infty$ limit. Hence the upper branch only exists in AdS. 


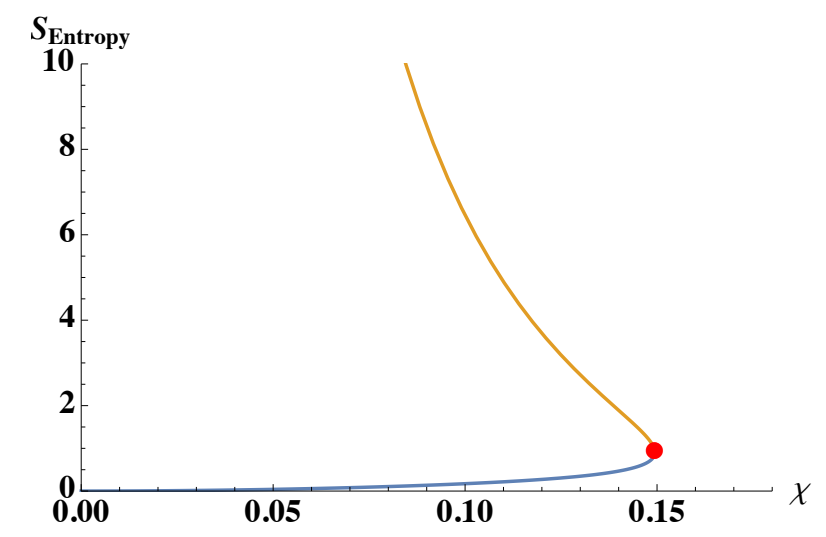

Figure 2. Entropy vs. NUT charge $\chi$ for uncharged TB-AdS for $\kappa=1$ and $G=l=1$.

From the renormalized action (2.26), we calculate thermodynamic energy, entropy, and specific heat using the definitions

$$
E=\partial_{\beta} I_{\text {ren. }}, \quad S=\left(\beta \frac{\partial}{\partial \beta}-1\right) I_{\text {ren. }}, \quad C=-\beta \frac{\partial S}{\partial \beta} .
$$

For $\kappa=1$, the energy and entropy become

$$
\begin{aligned}
& E_{(\kappa=1)}=\frac{\omega}{8 \pi G l^{2} r_{b}}\left(r_{b}^{4}-6 r_{b}^{2} \chi^{2}-3 \chi^{4}+l^{2}\left(r_{b}^{2}+\chi^{2}\right)\right)=\frac{\omega}{4 \pi G} M_{b}, \\
& S_{(\kappa=1)}=\frac{\omega}{4 G r_{b}}\left(r_{b}^{3}-r_{b} \chi^{2}+4 \kappa \chi^{3}-\frac{12 \chi^{3}\left(r_{b}^{2}+\chi^{2}\right)}{l^{2}}\right)=\frac{\omega \chi}{G}\left(M_{b}+\frac{r_{b}^{3}-3 r_{b} \chi^{2}}{l^{2}}\right)
\end{aligned}
$$

where the entropy is not the area of the TB-AdS horizon due to the contribution of Misner string. Inserting (2.29) into (2.34), we plot the entropy as a function of $\chi$ in figure 2 . We see that the entropy increases for the small TB-AdS (blue solid line) solution and decreases for the large one (orange solid line) as the NUT charge $\chi$ increases to its maximum value.

For $\kappa \neq 1$, the energy and entropy are

$$
\begin{aligned}
& E_{(\kappa \neq 1)}=\frac{\omega}{8 \pi G}\left(\kappa+\frac{r_{b}^{3}-3 r_{b} \chi^{2}}{l^{2}}\right), \\
& S_{(\kappa \neq 1)}=\frac{\omega}{4 G}\left(r_{b}^{2}-\chi^{2}\right)
\end{aligned}
$$

and the entropy agrees with the Bekenstein-Hawking formula. These quantities for all $\kappa$ satisfy the first law and free energy

$$
d E=T d S, \quad F=E-T S
$$

Finally, the heat capacity

$$
C=T \frac{\partial S}{\partial T}=T\left(\frac{\partial T}{\partial M} \frac{\partial M}{\partial r_{b}} \frac{\partial r_{b}}{\partial S}\right)^{-1}
$$




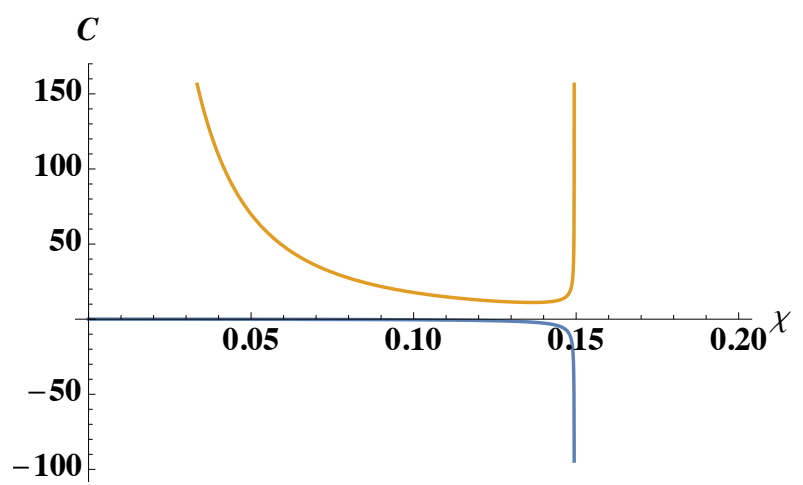

Figure 3. Heat Capacity (2.39) vs. NUT charge for uncharged TB-AdS for $\kappa=1$ and $G=l=1$.

is

$$
\begin{aligned}
C_{(\kappa=1)} & =\frac{\omega}{2 G} \frac{r_{b}\left(12 \chi^{3}-l^{2} r_{b}\right)}{\left(-12 r_{b} \chi+l^{2}\right)}, \\
C_{(\kappa \neq 1)} & =\frac{\omega r_{b}^{2}}{2 G}\left(\frac{3 r_{b}^{2}-3 \chi^{2}+\kappa l^{2}}{3 r_{b}^{2}+3 \chi^{2}-\kappa l^{2}}\right)
\end{aligned}
$$

for the different values of $\kappa$. We plot the $\kappa=1$ case as a function of $\chi$ (using (2.29)) in figure 3. Similar to small and large AdS black holes, the small (blue solid line) and large (orange solid line) TB-AdS solutions exhibit negative and positive heat capacity respectively.

\section{Thermodynamics of dyonic TN/TB-AdS}

To obtain dyonic Taub-NUT-AdS solutions, we add a gauge field to the action (2.1)

$$
I=\frac{1}{16 \pi G_{4}} \int d^{4} x \sqrt{-g}\left(R+\frac{6}{l^{2}}-F^{\mu \nu} F_{\mu \nu}\right)+\frac{1}{8 \pi G_{4}} \int d^{3} x \sqrt{-h}\left(K-\frac{2}{l}-\frac{l}{2} R_{3}\right)
$$

which leads to the equations of motion

$$
\begin{aligned}
R_{\mu \nu}-\frac{1}{2} g_{\mu \nu} R & =\frac{3}{l^{2}} g_{\mu \nu}+2\left(F_{\mu}{ }^{\sigma} F_{\nu \sigma}-\frac{1}{4} g_{\mu \nu} F_{\rho \sigma} F^{\rho \sigma}\right), \\
D_{\mu} F^{\mu \nu} & =0 .
\end{aligned}
$$

The solution for the metric and gauge field are

$$
\begin{aligned}
& d s^{2}=-f(r)(d t+2 s \lambda(\theta) d \phi)^{2}+\frac{d r^{2}}{f(r)}+\left(r^{2}+s^{2}\right)\left(d \theta^{2}+Y(\theta)^{2} d \phi^{2}\right), \\
& A=A_{\mu} d x^{\mu}=\frac{1}{2 s} h(r) d t+h(r) \lambda(\theta) d \phi
\end{aligned}
$$


where

$$
\begin{aligned}
& f(r)=\frac{l^{-2}\left(r^{2}+s^{2}\right)^{2}+\left(\kappa+4 l^{-2} s^{2}\right)\left(r^{2}-s^{2}\right)-2 M r+P^{2}+Q^{2}}{r^{2}+s^{2}}, \\
& h(r)=-\frac{2 Q s r+P\left(r^{2}-s^{2}\right)}{r^{2}+s^{2}} .
\end{aligned}
$$

From the field strength of the gauge field (3.5) we obtain the electric and magnetic charges

$$
\begin{aligned}
& Q_{e}\left[\xi_{t}\right] \equiv \frac{1}{\omega} \int_{\partial \Sigma_{\kappa}} * F=\lim _{r \rightarrow \infty} \frac{Q\left(r^{2}-s^{2}\right)-2 P r s}{r^{2}+s^{2}}=Q, \\
& Q_{m}\left[\xi_{\phi}\right] \equiv \frac{1}{\omega} \int_{\partial \Sigma_{\kappa}} F=\lim _{r \rightarrow \infty} \frac{P\left(r^{2}-s^{2}\right)+2 Q r s}{r^{2}+s^{2}}=P
\end{aligned}
$$

where $\Sigma_{\kappa}$ is the spacelike hypersurface for each $\kappa, \partial \Sigma_{\kappa}$ is the boundary of $\Sigma_{\kappa}$ at $r=$ constant, and the conserved charge is taken at $r=\infty$.

Once again, performing the Wick-rotation $t \rightarrow-i \tau$ and $s \rightarrow-i \chi$, we find

$$
I_{\text {ren. }}=-\frac{1}{16 \pi G_{4}} \int d^{4} x \sqrt{g}\left(R+\frac{6}{l^{2}}-F^{\mu \nu} F_{\mu \nu}\right)-\frac{1}{8 \pi G_{4}} \int d^{3} x \sqrt{h}\left(K-\frac{2}{l}-\frac{l}{2} R_{3}\right)
$$

for the Euclidean action. The metric and gauge field become

$$
\begin{aligned}
& d s_{\mathrm{E}}^{2}=f_{E}(r)(d \tau+2 \chi \lambda(\theta) d \phi)^{2}+\frac{d r^{2}}{f_{E}(r)}+\left(r^{2}-\chi^{2}\right)\left(d \theta^{2}+Y(\theta)^{2} d \phi^{2}\right) \\
& A_{\mathrm{E}}=\frac{1}{2 \chi} h_{E}(r) d \tau+h_{E}(r) \lambda(\theta) d \phi
\end{aligned}
$$

where

$$
\begin{aligned}
& f_{\mathrm{E}}=\frac{l^{-2}\left(r^{2}-\chi^{2}\right)^{2}+\left(\kappa-4 l^{-2} \chi^{2}\right)\left(r^{2}+\chi^{2}\right)-2 M r+P^{2}+Q^{2}}{r^{2}-\chi^{2}}, \\
& h_{\mathrm{E}}=\frac{2 i Q \chi r-P\left(r^{2}+\chi^{2}\right)}{r^{2}-\chi^{2}} .
\end{aligned}
$$

As with the uncharged case, this Euclidean metric describes two distinct geometries, a dyonic-TN-AdS and a dyonic-TB-AdS.

\subsection{Dyonic TN-AdS solution and thermodynamics}

The dyonic-TN-AdS solution is obtained when $f_{\mathrm{E}}(r)$ has a root at $r=r_{+}=\chi$. This implies

$$
M=M_{n}=\frac{1}{2 \chi}\left(2 \chi^{2}\left(\kappa-\frac{4 \chi^{2}}{l^{2}}\right)+P^{2}+Q^{2}\right) .
$$

and the Euclidean metric function is then given by

$$
f_{n}(r)=\frac{l^{-2}(r-\chi)^{2}(r+3 \chi) \chi+\kappa(r-\chi) \chi-\left(P^{2}+Q^{2}\right)}{\chi(r+\chi)} .
$$

It is clear that if $f_{n}(r=\chi)=0$, then

$$
P^{2}+Q^{2}=0
$$


We can satisfy this relation non-trivially if we take $P=i Q$ so as to make the gauge field vanish at the horizon, namely $h_{E}\left(r_{+}\right)=h(\chi)=0$. This implies

$$
h_{\mathrm{E}}(r)=-\frac{i Q(r-\chi)}{r+\chi} .
$$

Note that if (3.17) holds then the metric function becomes the same as the uncharged case, and so only $\kappa=0$ and 1 are allowed for dyonic TN-AdS. Imposing these conditions $r_{+}=\chi$ and $P=i Q$, the mass parameter becomes

$$
M_{n}=\chi \kappa-\frac{4 \chi^{3}}{l^{2}} .
$$

Since $K$ and $R_{3}$ are still given by (2.12) and (2.13) respectively, the on-shell Euclidean action is now

$$
\begin{gathered}
I_{\mathrm{E}}=\frac{\beta \omega}{8 \pi G}\left[3 M_{n}-2 \kappa R-\frac{2\left(R^{3}-4 R \chi^{2}-\chi^{3}\right)}{l^{2}}-\frac{Q^{2}}{2 \chi}+\mathcal{O}\left(\frac{1}{R}\right)\right]+I_{c t}, \\
I_{\text {ren. }}=\lim _{R \rightarrow \infty}\left(I_{\mathrm{E}}\right)=\frac{\beta \omega}{8 \pi G}\left(M_{n}+\frac{2 \chi^{3}}{l^{2}}-\frac{Q^{2}}{2 \chi}\right)=\frac{\beta \omega \chi}{8 \pi G}\left(\kappa-\frac{2 \chi^{2}}{l^{2}}-\frac{Q^{2}}{2 \chi^{2}}\right)
\end{gathered}
$$

where $\beta$ is the inverse of the Hawking temperature.

The thermodynamic energy, entropy, and specific heat are, for $\kappa=1$,

$$
\begin{aligned}
& S=\frac{\omega \chi^{2}}{G}\left(1-\frac{6 \chi^{2}}{l^{2}}+\frac{Q^{2}}{2 \chi^{2}}\right)=\frac{\omega \chi^{2}}{G}\left(1+2 g^{2}-\frac{6 \chi^{2}}{l^{2}}\right), \\
& E=\frac{\omega}{8 \pi G}\left(M_{n}+\chi \kappa-\frac{4 \chi^{3}}{l^{2}}+\frac{Q^{2}}{\chi}\right)=\frac{\omega \chi}{4 \pi G}\left(1+2 g^{2}-\frac{4 \chi^{2}}{l^{2}}\right), \\
& \Phi=\frac{i \omega}{4 \pi G} \frac{1}{2 \chi}\left(h_{\mathrm{E}}(\infty)-h_{\mathrm{E}}(\chi)\right)=-\frac{\omega}{4 \pi G} g .
\end{aligned}
$$

where we rescaled $Q=-2 g \chi$ (and so $P=-2 i g \chi$ ). The Hawking temperature is easily calculated to be

$$
T=\frac{1}{4 \pi} f_{n}^{\prime}(\chi)=\frac{\kappa}{8 \pi \chi}
$$

and we can verify that the first law and free energy are satisfied

$$
d E=T d S+\Phi d Q, \quad F=E-T S-\Phi Q .
$$

where $\chi$ and $g$ are the independent variables.

For $\kappa=0$ the temperature vanishes and we have an extremal solution. From the gauge potential, we expect the energy to be

$$
E=\frac{\omega}{4 \pi G}\left(\frac{Q^{2}}{4 \chi}-\frac{\chi^{3}}{l^{2}}\right), \quad \Phi=\frac{Q \omega}{8 \pi G \chi}
$$

so as to satisfy the following relations

$$
d E=\Phi d Q, \quad F=E-\Phi Q .
$$

Since the temperature is zero, we cannot determine the entropy using (2.18). However the horizon area is zero and the Misner string regularity condition does not apply, so we expect that the entropy is zero. 


\subsection{Dyonic TB-AdS}

If we assume that the metric (3.13) is not factored by $(r-\chi)$, then it has a two dimensional fixed point set and is called TB-AdS. The metric and gauge field are now

$$
\begin{aligned}
& f_{E}=\frac{l^{-2}\left(r^{2}-\chi^{2}\right)^{2}+\left(\kappa-4 l^{-2} \chi^{2}\right)\left(r^{2}+\chi^{2}\right)-2 M r+P^{2}+Q^{2}}{r^{2}-\chi^{2}}, \\
& h_{\mathrm{E}}=\frac{2 i Q \chi r-P\left(r^{2}+\chi^{2}\right)}{r^{2}-\chi^{2}} .
\end{aligned}
$$

and the mass parameter is

$$
M=M_{b}=\frac{l^{2}\left(P^{2}+Q^{2}+\kappa\left(r_{b}^{2}+\chi^{2}\right)\right)+r_{b}^{4}-6 r_{b}^{2} \chi^{2}-3 \chi^{4}}{2 l^{2} r_{b}}
$$

where we denote the radius of the dyonic-TB-AdS as $r_{b}$. Imposing the regularity condition for the norm of a gauge field to vanish at the horizon of TB-AdS, we get

$$
\left.g^{a b} A_{E a} A_{E b}\right|_{r=r_{b}}=A_{E}^{2}\left(r_{b}\right)=0 \rightarrow P=\frac{2 i Q \chi r_{b}}{r_{b}^{2}+\chi^{2}}
$$

and so $Q$ and $P$ are related.

We next calculate all thermodynamic quantities, subsequently apply the regularity condition, and then show the first law and free energy are satisfied. To calculate the electric and magnetic potentials, we consider two definitions of the field potential as follows

$$
\Phi_{E}^{(1)}=\left.\frac{\partial E}{\partial Q}\right|_{r_{b}, P}-\left.T \frac{\partial S}{\partial Q}\right|_{r_{b}, P}, \quad \Phi_{M}^{(1)}=\left.\frac{\partial E}{\partial P}\right|_{r_{b}, Q}-\left.T \frac{\partial S}{\partial P}\right|_{r_{b}, Q}
$$

where $\Phi_{E}^{(1)}$ and $\Phi_{M}^{(1)}$ are conjugate variables for each charges $Q$ and $P$ respectively via the first law of thermodynamics

$$
d E=T d S+\Phi_{E} d Q+\Phi_{M} d P
$$

with details about how to compute them given in appendix B. Alternatively, we can take

$$
\Phi_{E}^{(2)}=\frac{i \omega}{4 \pi G}\left(A_{\tau}(\infty)-A_{\tau}\left(r_{b}\right)\right), \quad \Phi_{M}^{(2)}=\frac{\omega}{4 \pi G}\left(\Phi_{M}(\infty)-\Phi_{M}\left(r_{b}\right)\right)
$$

which are typically chosen for gauge potentials, and where

$$
\Phi_{M}(r)=\int^{r} d r^{\prime} B\left(r^{\prime}\right), \quad B(r)=\frac{1}{\sqrt{g}} \epsilon^{t r \theta \phi} F_{\theta \phi} .
$$

Then we can compute energy from the equation

$$
E-\Phi_{\mathrm{E}} Q-\Phi_{\mathrm{M}} P=\partial_{\beta} I_{\mathrm{ren}}
$$

for both cases.

Since we have a dyonic solution we naturally expect both types of charge to appear in the first law, and usually they yield the same result for an electric potential, as shown 
in appendix A. Here, however, this turns out not to be the case: upon computing the potentials respectively using (3.31) and (3.33) for each case and then imposing the regularity condition (3.30), we find that thermodynamic quantities such as energy, temperature, entropy, and electric potentials $\Phi_{E}^{(1)}$ and $\Phi_{E}^{(2)}$ become equivalent, whereas the magnetic potentials $\Phi_{M}^{(1)}$ and $\Phi_{M}^{(2)}$ remain inequivalent. This is because the regularity condition is equivalent to setting the magnetic potential $\Phi_{M}^{(1)}=0$, a condition incompatible with $\Phi_{M}^{(2)}$ in (3.33). Thus the magnetic potential $\Phi_{M}^{(2)}$ is not valid once the regularity condition (3.30) is imposed. Then under this regularity condition (3.30) we expect the first law and free energy to satisfy

$$
d E=T d S+\Phi_{E} d Q, \quad F=E-T S-\Phi_{E} Q
$$

and we shall illustrate this in specific cases in what follows. In general only $\Phi_{M}^{(1)}$ can satisfy the first law, as should be clear from its definition in (3.31).

\subsubsection{Thermodynamics for $\kappa \neq 1$}

The extrinsic curvature and three dimensional Ricci scalar are

$$
\begin{aligned}
K= & \frac{-3 M_{b} r^{2}+M_{b} \chi^{2}+2 \kappa r^{3}+r\left(P^{2}+Q^{2}\right)+\left(3 r^{5}-14 r^{3} \chi^{2}+3 r \chi^{4}\right) l^{-2}}{\left(r^{2}-\chi^{2}\right)^{2} \sqrt{f_{\mathrm{E}}(r)}} \\
R_{3}= & \frac{-2 \chi^{2}\left(-2 M_{b} r+P^{2}+Q^{2}+\kappa\left(r^{2}+\chi^{2}\right)+\left(r^{4}-6 r^{2} \chi^{2}-3 \chi^{4}\right) l^{-2}\right) \lambda^{\prime}(\theta)^{2}}{\left(r^{2}-\chi^{2}\right)^{3} Y(\theta)^{2}} \\
& -\frac{2 Y^{\prime \prime}(\theta)}{\left(r^{2}-\chi^{2}\right) Y(\theta)}
\end{aligned}
$$

and from this we obtain

$$
\begin{aligned}
I_{\mathrm{E}}= & \frac{\beta \omega}{8 \pi G}\left[3 M_{b}-2 \kappa R-\frac{\left(2 R^{3}-8 R \chi^{2}+r_{b}^{3}-3 r_{b} \chi^{2}\right)}{l^{2}}\right. \\
& \left.-\frac{r_{b}\left(\left(Q^{2}-P^{2}\right)\left(r_{b}^{2}+\chi^{2}\right)+4 i P Q r_{b} \chi\right)}{\left(r_{b}^{2}-\chi^{2}\right)^{2}}+\mathcal{O}\left(\frac{1}{R}\right)\right]+I_{\mathrm{ct}} \\
I_{\mathrm{ren}}= & \lim _{R \rightarrow \infty}\left(I_{\mathrm{E}}\right)=\frac{\beta \omega}{8 \pi G}\left(M_{b}-\frac{r_{b}^{3}-3 r_{b} \chi^{2}}{l^{2}}+\frac{\left(P^{2}-Q^{2}\right)\left(r_{b}^{2}+\chi^{2}\right) r_{b}-4 i P Q r_{b}^{2} \chi}{\left(r_{b}^{2}-\chi^{2}\right)^{2}}\right) .
\end{aligned}
$$

for the Euclidean action. Note from (3.30) that $P \propto-i 2 Q \chi$, ensuring the Euclidean action (3.40) is real.

The Hawking temperature and entropy are

$$
\begin{aligned}
T & =\frac{1}{4 \pi} f_{E}^{\prime}\left(r_{b}\right) \\
& =\frac{r_{b} \chi^{2}}{\pi\left(r_{b}^{2}-\chi^{2}\right)^{2}}\left(\frac{M_{b}\left(r_{b}^{2}+\chi^{2}\right)}{2 r_{b} \chi^{2}}+\frac{r_{b}^{4}-2 r_{b}^{2} \chi^{2}+9 \chi^{4}}{2 l^{2} \chi^{2}}-\frac{\left(P^{2}+Q^{2}\right)}{2 \chi^{2}}-\kappa\right) \\
& =\frac{l^{2}\left(\kappa r_{b}-M_{b}\right)+2 r_{b}\left(r_{b}^{2}-3 \chi^{2}\right)}{2 \pi l^{2}\left(r_{b}^{2}-\chi^{2}\right)} \\
S & \equiv\left(\beta \frac{\partial}{\partial \beta}-1\right) I_{\text {ren. }}=\frac{\omega\left(r_{b}^{2}-\chi^{2}\right)}{4 G},
\end{aligned}
$$


where

$$
\beta=\frac{2 \pi l^{2}\left(r_{b}^{2}-\chi^{2}\right)}{l^{2}\left(\kappa r_{b}-M_{b}\right)+2 r_{b}\left(r_{b}^{2}-3 \chi^{2}\right)}
$$

allows us to regard $M_{b}$ as a function of $\beta$ and the other parameters. The heat capacity for fixed $P$ and $Q$ is

$$
C_{P, Q}=\frac{\omega r_{b}^{2}\left(r_{b}^{2}-\chi^{2}\right)\left(3\left(r_{b}^{2}-\chi^{2}\right)^{2}-l^{2}\left(-\kappa r_{b}^{2}+\kappa \chi^{2}+P^{2}+Q^{2}\right)\right)}{2 G\left(l^{2}\left(\left(P^{2}+Q^{2}\right)\left(3 r_{b}^{2}-\chi^{2}\right)-\kappa\left(r_{b}^{2}-\chi^{2}\right)^{2}\right)+3\left(r_{b}^{2}-\chi^{2}\right)^{2}\left(r_{b}^{2}+\chi^{2}\right)\right)}
$$

from (2.38). Now we obtain the electric, magnetic potentials and energy

$$
\begin{aligned}
\Phi_{\mathrm{E}}^{(1)} & =\frac{\omega}{4 \pi G} \frac{Q\left(r_{b}^{2}+\chi^{2}\right) r_{b}+2 i P r_{b}^{2} \chi}{\left(r_{b}^{2}-\chi^{2}\right)^{2}}, \quad \Phi_{\mathrm{M}}^{(1)}=\frac{\omega}{4 \pi G} \frac{2 i Q r_{b}^{2} \chi-P\left(r_{b}^{2}+\chi^{2}\right) r_{b}}{\left(r_{b}^{2}-\chi^{2}\right)^{2}} \\
E^{(1)} & =\frac{r_{b} \omega}{8 \pi G}\left(\kappa+\frac{r_{b}^{2}-3 \chi^{2}}{l^{2}}+\frac{\left(Q^{2}-P^{2}\right)\left(r_{b}^{2}+\chi^{2}\right)+4 i P Q r_{b} \chi}{\left(r_{b}^{2}-\chi^{2}\right)^{2}}\right)
\end{aligned}
$$

where $\Phi_{\mathrm{E}}^{(1)}$ and $\Phi_{\mathrm{M}}^{(1)}$ are derived from (3.31) and the energy is from (3.35), and

$$
\begin{aligned}
\Phi_{\mathrm{E}}^{(2)} & =\frac{\omega}{4 \pi G} \frac{Q r_{b}+i P \chi}{\left(r_{b}^{2}-\chi^{2}\right)}, \quad \Phi_{\mathrm{M}}^{(2)}=\frac{\omega}{4 \pi G} \frac{i Q \chi-P r_{b}}{\left(r_{b}^{2}-\chi^{2}\right)} \\
E^{(2)} & =\frac{\omega}{8 \pi G}\left(\kappa r_{b}+\frac{r_{b}^{3}-3 r_{b} \chi^{2}}{l^{2}}+\frac{\left(P^{2} r_{b}-2 i P Q \chi\right)\left(r_{b}^{2}+\chi^{2}\right)+Q^{2} r_{b}\left(r_{b}^{2}-3 \chi^{2}\right)}{\left(r_{b}^{2}-\chi^{2}\right)^{2}}\right) .
\end{aligned}
$$

where $\Phi_{\mathrm{E}}^{(2)}$ and $\Phi_{\mathrm{M}}^{(2)}$ are derived from (3.33) and the energy is computed from (3.35). These quantities do not satisfy the first law of thermodynamics, but upon imposing the regularity condition (3.30) these thermodynamic variables (3.45)-(3.48) become

$$
\begin{aligned}
& E^{(1)}=E^{(2)}=E=\frac{\omega}{8 \pi G}\left(\kappa r_{b}+\frac{r_{b}\left(r_{b}^{2}-3 \chi^{2}\right)}{l^{2}}+\frac{Q^{2} r_{b}}{r_{b}^{2}+\chi^{2}}\right) \\
& \Phi_{\mathrm{E}}^{(1)}=\Phi_{\mathrm{E}}^{(2)}=\Phi_{\mathrm{E}}=\frac{\omega}{4 \pi G} \frac{Q r_{b}}{\left(r_{b}^{2}+\chi^{2}\right)} \quad \Phi_{\mathrm{M}}^{(1)}=0 \neq \Phi_{\mathrm{M}}^{(2)}=-\frac{\omega}{4 \pi G} \frac{i Q \chi}{\left(r_{b}^{2}+\chi^{2}\right)}
\end{aligned}
$$

and we see that the magnetic potentials are inequivalent. The first law and the free energy become

$$
\begin{aligned}
d E & =T d S+\Phi_{\mathrm{E}} d Q, \\
F & =E-T S-\Phi_{\mathrm{E}} Q
\end{aligned}
$$

upon using $\Phi_{\mathrm{M}}^{(1)}=\Phi_{\mathrm{M}}=0$.

\subsubsection{Thermodynamics for $\kappa=1$}

For $\kappa=1$, the Hawking temperature is constrained to be $1 / 8 \pi \chi$ so that the Misner string singularity is no longer present. Moreover, the entropy does not follow the Bekenstein- 
Hawking formula, but can be obtained from the thermodynamic relation (2.32). We find

$$
\begin{aligned}
T & =\frac{1}{4 \pi} f_{E}^{\prime}\left(r_{b}\right) \\
& =\frac{r_{b} \chi^{2}}{\pi\left(r_{b}^{2}-\chi^{2}\right)^{2}}\left(\frac{M_{b}\left(r_{b}^{2}+\chi^{2}\right)}{2 r_{b} \chi^{2}}+\frac{r_{b}^{4}-2 r_{b}^{2} \chi^{2}+9 \chi^{4}}{2 l^{2} \chi^{2}}-\frac{\left(P^{2}+Q^{2}\right)}{2 \chi^{2}}-1\right)=\frac{1}{8 \pi \chi}, \\
S & \equiv\left(\beta \frac{\partial}{\partial \beta}-1\right) I_{\mathrm{E}}=\left(\chi \frac{\partial}{\partial \chi}-1\right) I_{\mathrm{E}} \\
& =\frac{\omega}{2 G}\left(\frac{l^{2}\left(r_{b}^{2}+\chi^{2}\right)-24 r_{b} \chi^{3}}{2 l^{2}}+\frac{4 r_{b} \chi^{2}\left(\left(P^{2}-Q^{2}\right)\left(3 r_{b}^{2} \chi+\chi^{3}\right)-2 i P Q r_{b}\left(r_{b}^{2}+3 \chi^{2}\right)\right)}{\left(r_{b}^{2}-\chi^{2}\right)^{3}}\right) .
\end{aligned}
$$

without imposing (3.30). We shall first consider the behaviour of these quantities without this constraint, and later see that it is required in order that the first law of thermodynamics hold.

Inserting $M_{b}$ from (3.29) into the temperature yields the constraint

$$
Q^{2}+P^{2}=\frac{\left(r_{b}^{2}-\chi^{2}\right)\left(6 \chi\left(r_{b}^{2}-\chi^{2}\right)-l^{2}\left(r_{b}-2 \kappa \chi\right)\right)}{2 l^{2} \chi} .
$$

Replacing $P=-2 i \chi g$, we can interpret this as a constraint on $r_{b}$. Then (3.57) has 4 solutions denoted $r_{b, 1 \mp}$ and $r_{b, 2 \mp}$, each of which are functions of $Q, g$ and $\chi$. We write these in appendix C, and plot each as a function of $\chi$ in figure 4 for fixed values of $Q$ and $g$.

The quantities $r_{b, 1+}$ and $r_{b, 2+}$ are always positive, whereas the positivity of $r_{b, 1-}$ and $r_{b, 2-}$ depends on the parameter values; all positive values of $r_{b, 1-}$ yield negative entropy, but some positive values of $r_{b, 2-}$ can yield positive entropy. Hence we shall only consider $r_{b, 1+}, r_{b, 2+}$, and $r_{b, 2-}$ in this section.

In the uncharged case as shown in figure $1, r_{b}$ in the small TB-AdS started at $\chi=0$; upon turning on the electric charge $Q$ with the magnetic parameter $g=0$, the starting point is at a positive value of $\chi$, illustrated in the upper left plot in figure 4. As $Q$ increases the curve approaches an inflection point and retains the small/large branch shape for a while. Further increasing $Q$, the range of $r_{b}$ of the small TB-AdS gets shorter and disappears at around $Q=0.13$. However another solution of $r_{b, 1+}$ at large values of $\chi$ emerges, extending the end point of $r_{b}$ of the large TB-AdS; this solution is denoted with the orange dashed line in the upper right plot in figure 4 . All dashed lines in figure 4 correspond to negative entropy, and so are not considered to be physical solutions. Thus as the electric charge $Q$ becomes larger than $\sim 0.13$ (with $g=0$ ) only the large (dyonic) TB-AdS solution exists.

Once the magnetic parameter $g$ becomes nonzero, the bolt radius $r_{b}$ of the small TB-AdS appears and again forms the small/large branches, shown in the lower left plot in figure 4. Setting $Q=0$, we recover the behaviour of the uncharged case; note that $P=-2 i g \chi$ and so the pure magnetic case does not exhibit behaviour similar to the pure electric case. If we increase $Q$ the small branch disappears (from the upper left to the upper right plot in figure 4 , but if we increase $g$, then for $g>Q$ the small branch emerges again (the lower left plot becomes the lower right one). 

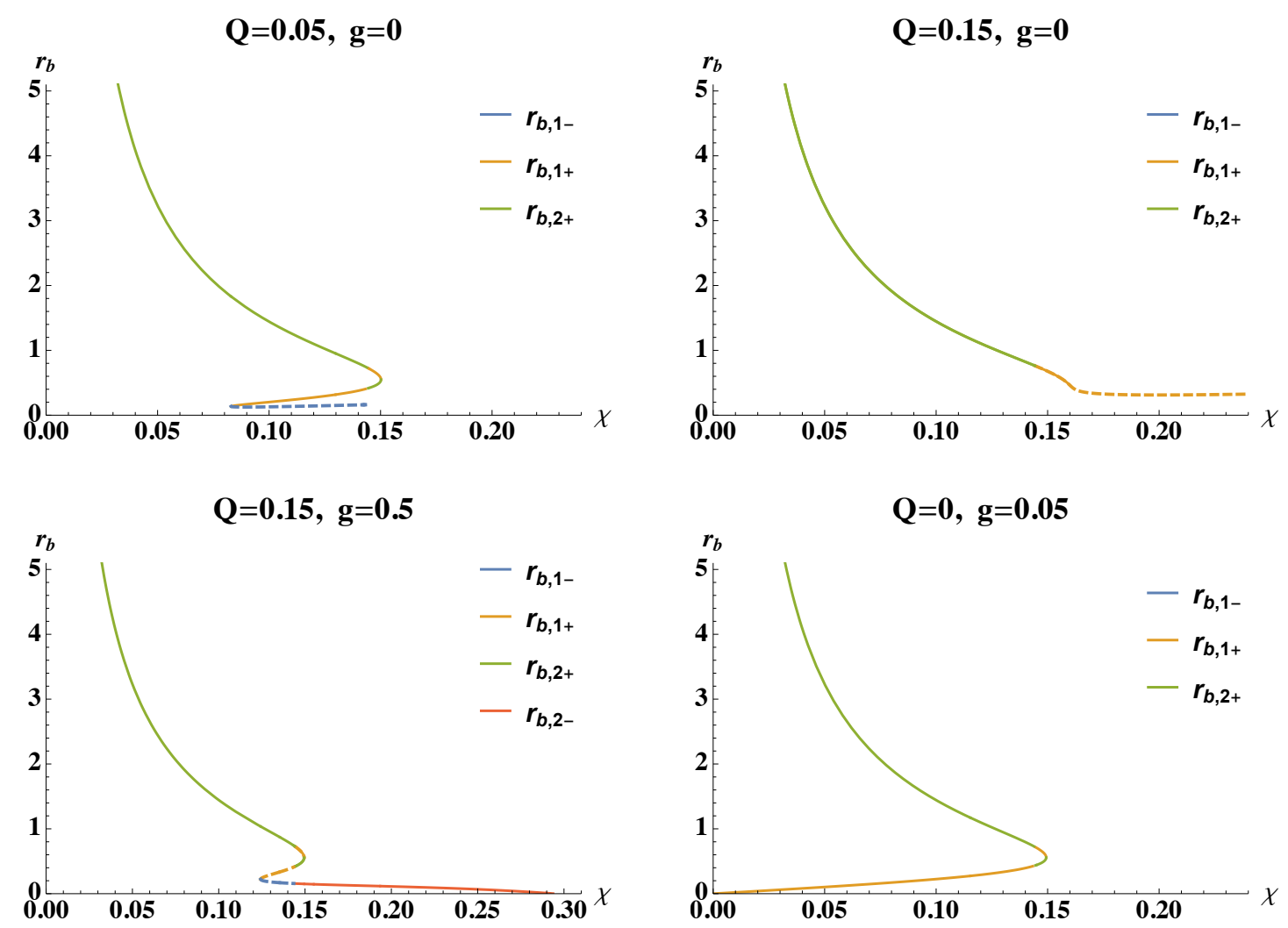

Figure 4. The horizon radius of TB-AdS for $\kappa=1$ vs. $\chi$ for fixed values of $Q$ and $g$ with $G=l=1$. The dashed lines correspond to negative entropy, and are not regarded as corresponding to physically admissible solutions.

In general we find that a maximum value of $\chi$ always exists. The dyonic TB-AdS solution has two branches (large and small) for two cases: $Q<0.13$ with $g=0$, and $g>Q$. Recall that a small (dyonic) TB-AdS is one whose radius increases as the NUT charge increases, whereas the radius of a large (dyonic) TB-AdS decreases as the NUT charge increases.

Inserting these values of $r_{b}$ into the entropy (3.56), we can express $S$ in terms of $Q, g$ and $\chi$. For fixed values of $Q$ and $g$, the entropy as a function of $\chi$ is plotted in figure 5 where the dashed lines are ones calculated with the dashed lines of $r_{b, 1+}$ in figure 4 . We see that even for the $r_{b,+}$ solutions, the parameter range must be restricted to ensure positive entropy. We also observe that if $Q$ is smaller than 0.13 with $g=0$ or $g$ is bigger than $Q$, the behaviour of the entropy becomes similar to the uncharged TB-AdS case, shown in figure 2, but as $Q$ increases keeping $g=0$ the only allowed solution is that of the large (dyonic) TB-AdS.

From the definition (2.38), we also calculate the heat capacity

$$
\begin{aligned}
C_{P, Q}= & \frac{\omega l^{2} r_{b} \chi^{2}}{2 G a_{1}}\left(\frac{\left(r_{b}^{2}-\chi^{2}\right)^{2}\left(l^{2} r_{b}-12 \chi^{3}\right)}{l^{2} \chi^{2}}\right. \\
& \left.+\frac{4 \chi\left(Q^{2}-P^{2}\right)\left(9 r_{b}^{4}+14 r_{b}^{2} \chi^{2}+\chi^{4}\right)+16 i P Q r_{b}\left(r_{b}^{4}+8 r_{b}^{2} \chi^{2}+3 \chi^{4}\right)}{\left(r_{b}^{2}-\chi^{2}\right)^{2}}\right)
\end{aligned}
$$



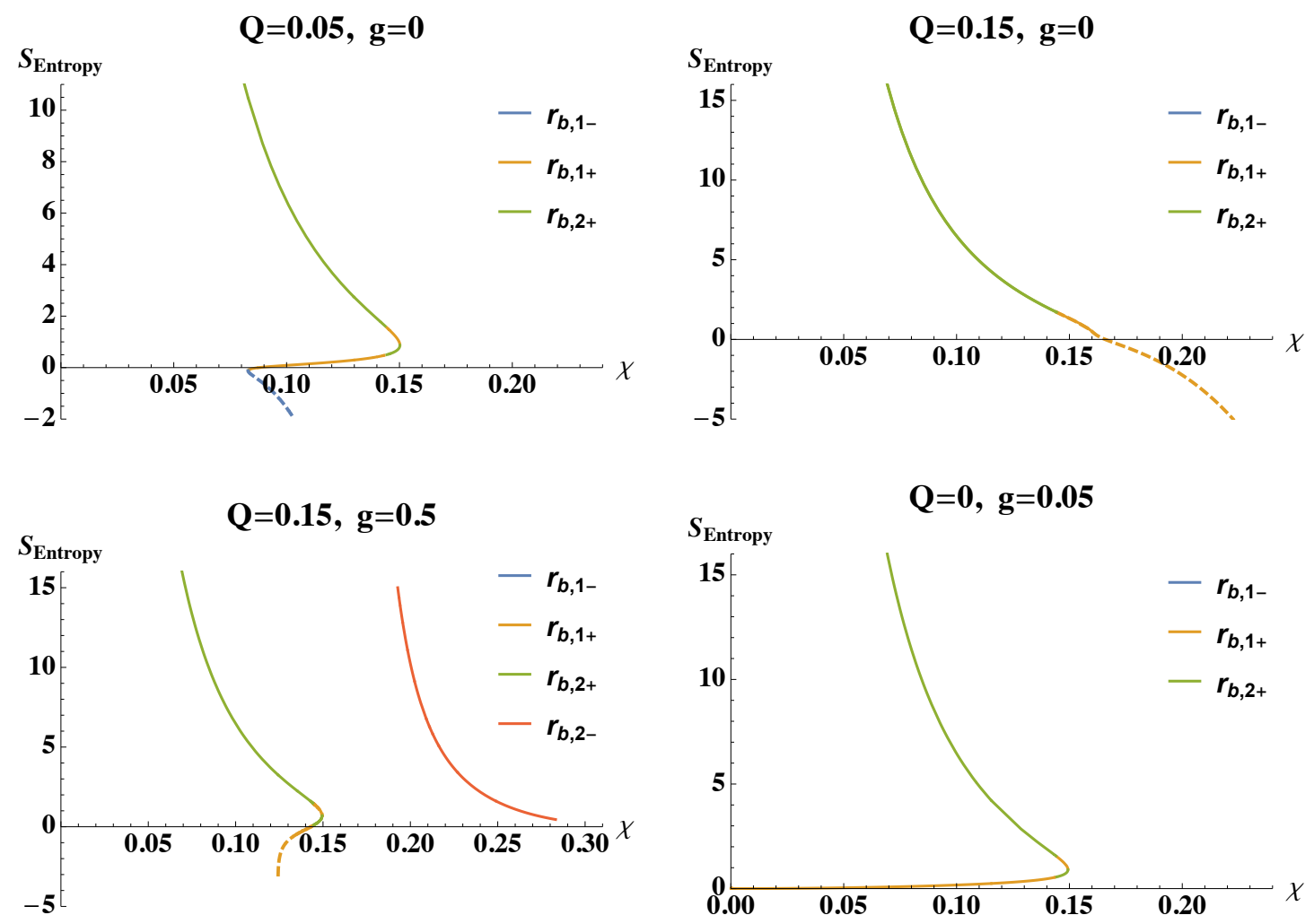

Figure 5. Entropy (3.56) of TB-AdS for $\kappa=1$ vs. $\chi$ for fixed values of $Q$ and $g$ with $G=l=1$.

for fixed $P$ and $Q$, where

$$
a_{1}=12 \chi r_{b}\left(r_{b}^{2}-\chi^{2}\right)^{2}+l^{2}\left(2 \chi^{2} r_{b}^{2}-r_{b}^{4}-\chi^{4}+4 \chi r_{b}\left(P^{2}+Q^{2}\right)\right) .
$$

Substituting $r_{b, 1+}$ and $r_{b, 2+}$, we plot the heat capacity (3.58) as a function of $\chi$ in figure 6 . If $Q<0.13$ with $g=0$ or as $g$ becomes larger than $Q$, the heat capacity exhibits behaviour similar to the uncharged TB-AdS case, shown in figure 3; the small (dyonic) TB-AdS has negative heat capacity and the large (dyonic) TB-AdS has positive heat capacity. Other cases, for which $r_{b, 1+}$ and $r_{b, 2+}$ are restricted to yield positive entropy, have only positive heat capacity, shown in the upper right graph of figure 6 .

From the Euclidean action, the energy, electric, and magnetic potentials are obtained from (3.31) and (3.35)

$$
\begin{aligned}
& \Phi_{\mathrm{E}}^{(1)}=\frac{\omega}{4 \pi G} \frac{r_{b}\left(2 i P \chi r_{b}+Q\left(r_{b}^{2}+\chi^{2}\right)\right)}{\left(\chi^{2}-r_{b}^{2}\right)^{2}}, \\
& \Phi_{\mathrm{M}}^{(1)}=-\frac{\omega}{4 \pi G} \frac{r_{b}\left(P\left(r_{b}^{2}+\chi^{2}\right)-2 i Q \chi r_{b}\right)}{\left(\chi^{2}-r_{b}^{2}\right)^{2}} . \\
& E^{(1)}=\frac{\omega}{8 \pi G}\left[M_{b}+\frac{r_{b}^{2}+\chi^{2}}{4 \chi}-\frac{3 \chi^{2} r_{b}+r_{b}^{3}}{l^{2}}\right. \\
& \left.+\frac{\left(Q^{2}-P^{2}\right)\left(-3 \chi^{4} r_{b}-6 \chi^{2} r_{b}^{3}+r_{b}^{5}\right)}{\left(r_{b}^{2}-\chi^{2}\right)^{3}}+\frac{16 i P Q \chi^{3} r_{b}^{2}}{\left(\chi^{2}-r_{b}^{2}\right)^{3}}\right],
\end{aligned}
$$



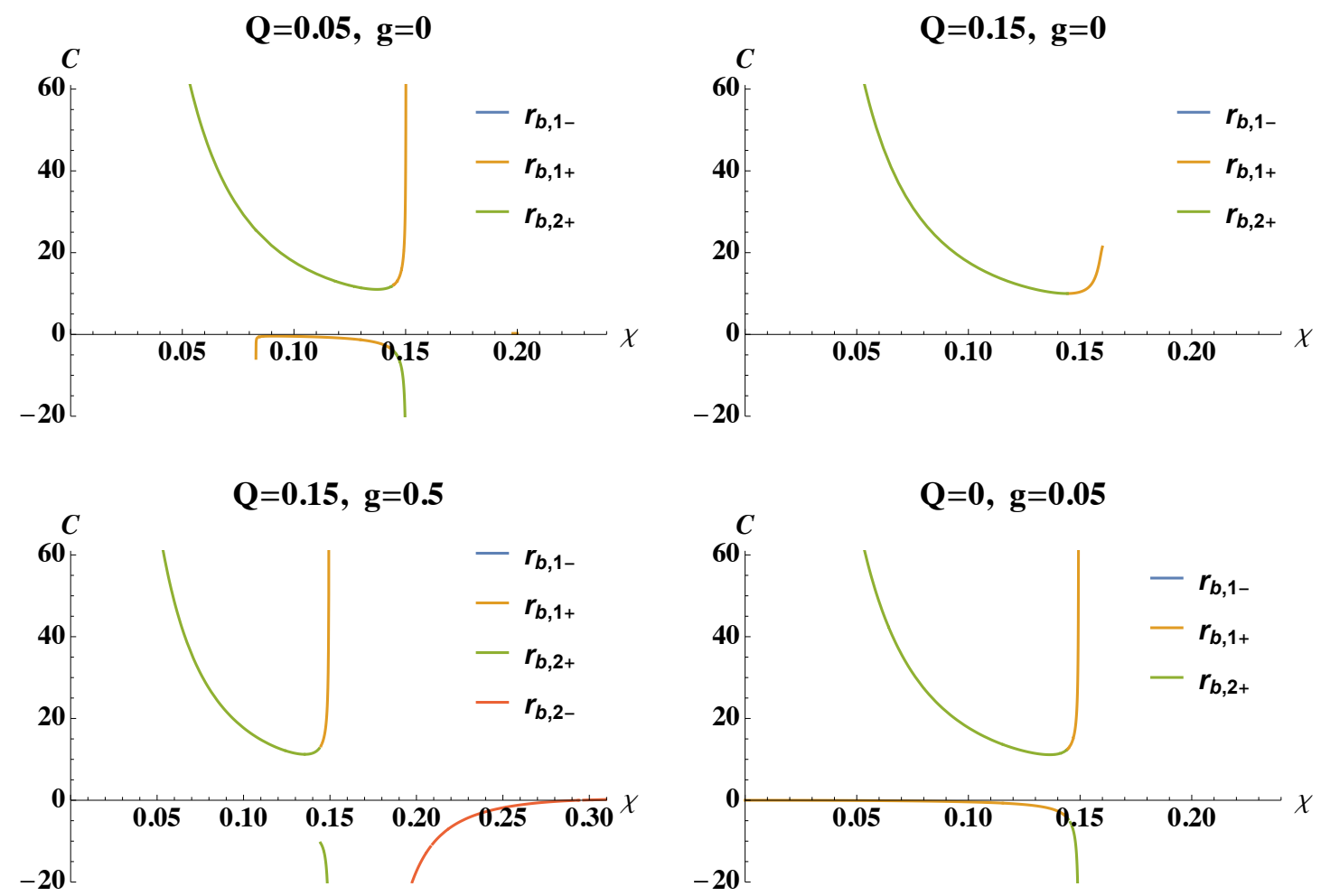

Figure 6. Heat capacity (3.58) for TB-AdS for $\kappa=1$ vs. $\chi$ for fixed $Q$ and $g$ with $G=l=1$.

and by (3.33) and (3.35)

$$
\begin{aligned}
\Phi_{\mathrm{E}}^{(2)}= & \frac{\omega}{4 \pi G} \frac{\left(Q r_{b}+i P \chi\right)}{\left(r_{b}^{2}-\chi^{2}\right)}, \quad \Phi_{\mathrm{M}}^{(2)}=\frac{\omega}{4 \pi G} \frac{\left(-P r_{b}+i Q \chi\right)}{\left(r_{b}^{2}-\chi^{2}\right)} \\
E^{(2)}= & \frac{\omega}{8 \pi G}\left[M_{b}+\frac{r_{b}^{2}+\chi^{2}}{4 \chi}-\frac{r_{b}\left(r_{b}^{2}+3 \chi^{2}\right)}{l^{2}}+\frac{1}{\left(r_{b}^{2}-\chi^{2}\right)^{3}}\left(P^{2} r_{b}\left(r_{b}^{4}+6 r_{b}^{2} \chi^{2}+\chi^{4}\right)\right.\right. \\
& \left.\left.+2 i P Q \chi\left(-3 r_{b}^{4}-6 r_{b}^{2} \chi^{2}+\chi^{4}\right)+Q^{2} r_{b}\left(r_{b}^{4}-10 r_{b}^{2} \chi^{2}+\chi^{4}\right)\right)\right]
\end{aligned}
$$

As stated above, we find that the first law cannot be satisfied unless the regularity condition (3.30) is imposed. Upon doing this, we find that (3.61)-(3.62) and (3.56) become

$$
\begin{aligned}
E & =\frac{\omega}{8 \pi G}\left[M_{b}+\frac{r_{b}^{2}+\chi^{2}}{4 \chi}-\frac{r_{b}\left(r_{b}^{2}+3 \chi^{2}\right)}{l^{2}}+\frac{Q^{2}\left(3 \chi^{2} r_{b}+r_{b}^{3}\right)}{\left(r_{b}^{2}+\chi^{2}\right)^{2}}\right], \\
S & =\frac{\omega}{4 G}\left[r_{b}^{2}+\chi^{2}-\frac{24 \chi^{3} r_{b}}{l^{2}}+\frac{8 Q^{2} \chi^{3} r_{b}}{\left(r_{b}^{2}+\chi^{2}\right)^{2}}\right], \\
\Phi_{\mathrm{E}} & =\frac{\omega}{4 \pi G} \frac{Q r_{b}}{\left(r_{b}^{2}+\chi^{2}\right)}, \quad \Phi_{\mathrm{M}}=0
\end{aligned}
$$

and that these quantities satisfy the first law

$$
\begin{aligned}
d E & =T d S+\Phi_{\mathrm{E}} d Q \\
F & =E-T S-\Phi_{\mathrm{E}} Q
\end{aligned}
$$

with the free energy written as in (3.68). Note that $g$ and $Q$ are no longer independent. 


\section{Zero temperature limit of dyonic TB-AdS}

For vanishing Hawking temperature the TB-AdS metric (3.13) factorizes:

$$
f_{\mathrm{E}}=\frac{1}{l^{2}\left(r^{2}-\chi^{2}\right)}\left(r+r_{0}+\alpha\right)\left(r+r_{0}-\alpha\right)\left(r-r_{0}\right)^{2}
$$

where

$$
\begin{aligned}
r_{0} & =\sqrt{\chi^{2}+\frac{1}{6} l\left(\sqrt{\kappa^{2} l^{2}+12\left(Q^{2}+P^{2}\right)}-\kappa l\right)}, \\
\alpha & =\sqrt{4 \chi^{2}-\frac{1}{3} l\left(\sqrt{\kappa^{2} l^{2}+12\left(Q^{2}+P^{2}\right)}+2 \kappa l\right)} .
\end{aligned}
$$

By writing

$$
\tau \rightarrow \frac{\tilde{\tau}}{\epsilon}, \quad r \rightarrow r_{0}+\epsilon \tilde{r}
$$

the metric (3.11) with $f_{E}$ given by (4.1) becomes

$$
d s_{\mathrm{ext}}^{2} \sim \frac{\tilde{r}^{2} f_{\epsilon,-}}{l^{2}\left(r_{0}^{2}-x^{2}\right)^{2}}(d \tilde{\tau}+2 \chi \lambda(\theta) \epsilon d \phi)^{2}+\frac{l^{2} r_{0} f_{\epsilon,+}}{\tilde{r}^{2}\left(4 r_{0}^{2}-\alpha^{2}\right)^{2}} d \tilde{r}^{2}+\left(r_{0}^{2}-\chi^{2}+2 \tilde{r} r_{0} \epsilon\right) d \Omega_{\kappa}
$$

to leading order in $\epsilon$. In the limit $\epsilon \rightarrow 0$ we obtain the near-horizon metric

$$
d s_{\mathrm{ext}}^{2} \rightarrow \frac{\tilde{r}^{2}}{l^{2}} \frac{\left(4 r_{0}^{2}-\alpha^{2}\right)}{\left(r_{0}^{2}-\chi^{2}\right)} d \tilde{\tau}^{2}+\frac{l^{2}}{\tilde{r}^{2}} \frac{\left(r_{0}^{2}-\chi^{2}\right)}{\left(4 r_{0}^{2}-\alpha^{2}\right)} d \tilde{r}^{2}+\left(r_{0}^{2}-\chi^{2}\right)\left(d \theta^{2}+Y(\theta)^{2} d \phi^{2}\right)
$$

which is $A d S_{2} \times \mathcal{M}_{\kappa}$, where $\mathcal{M}_{\kappa}$ is a two dimensional manifold depending on $\kappa$ as given by $(2.5)$, and where

$$
\begin{aligned}
4 r_{0}^{2}-\alpha^{2} & =l \sqrt{\kappa^{2} l^{2}+12\left(Q^{2}+P^{2}\right)} \\
r_{0}^{2}-\chi^{2} & =\frac{l}{6} \sqrt{\kappa^{2} l^{2}+12\left(Q^{2}+P^{2}\right)}-\frac{\kappa}{6} l^{2}
\end{aligned}
$$

It is interesting to note that no NUT charge $\chi$ appears in the $A d S_{2}$ section due to the relations (4.7), and the metric (4.6) has no singularities at the horizon.

Thus for $\kappa \neq 1$ the extremal limit is given by taking the metric function to be (4.1) and we can check if the thermodynamic relations hold in this limit. The Hawking temperature (3.41) is

$$
T_{\mathrm{H}}=\frac{3\left(r_{b}^{2}-\chi^{2}\right)^{2}+l^{2}\left(-Q^{2}-P^{2}+\left(r_{b}^{2}-\chi^{2}\right) \kappa\right)}{4 \pi r_{b} l^{2}\left(r_{b}^{2}-\chi^{2}\right)}
$$

upon substituting for the mass parameter. At zero temperature the following condition

$$
Q_{\mathrm{ext}}^{2}+P_{\mathrm{ext}}^{2}=\frac{\left(r_{b}^{2}-\chi^{2}\right)\left(3 r_{b}^{2}-3 \chi^{2}+\kappa l^{2}\right)}{l^{2}}
$$


must hold, implying that

$$
\begin{aligned}
M & =M_{\mathrm{ext}}=\frac{r_{b}\left(2 r_{b}^{2}-6 \chi^{2}+\kappa l^{2}\right)}{l^{2}} \\
& =\frac{\left(2 \kappa l^{2}-12 \chi^{2}+l \sqrt{\kappa^{2} l^{2}+12\left(Q_{\mathrm{ext}}^{2}+P_{\mathrm{ext}}^{2}\right)}\right) \sqrt{6 \chi^{2}-l^{2} \kappa+l \sqrt{\kappa^{2} l^{2}+12\left(Q_{\mathrm{ext}}^{2}+P_{\mathrm{ext}}^{2}\right)}}}{3 \sqrt{6} l^{2}} .
\end{aligned}
$$

as well as

$$
Q_{\mathrm{ext}}^{2}=\left(r_{b}^{2}+\chi^{2}\right)^{2}\left(\frac{\kappa}{r_{b}^{2}-\chi^{2}}+\frac{3}{l^{2}}\right)
$$

upon imposing the regularity condition.

The free energy and the energy (3.49) become

$$
F_{\mathrm{ext}}=-\frac{\omega r_{b}}{4 \pi G}\left(\frac{\kappa \chi^{2}}{r_{b}^{2}-\chi^{2}}+\frac{r_{b}^{2}+3 \chi^{2}}{l^{2}}\right), \quad E_{\mathrm{ext}}=\frac{\omega r_{b}^{3}}{4 \pi G}\left(\frac{\kappa}{r_{b}^{2}-\chi^{2}}+\frac{2}{l^{2}}\right) .
$$

This satisfies the following relation

$$
\begin{aligned}
F_{\text {ext }} & =E_{\text {ext }}-\left.\Phi_{\mathrm{E}} Q\right|_{\text {ext }}, \\
d E_{\text {ext }} & =\left.\Phi_{\mathrm{E}} d Q\right|_{\text {ext }}
\end{aligned}
$$

where we used $\Phi_{\mathrm{E}}$ in (3.66) with $Q=Q_{\text {ext }}$

$$
\Phi_{\mathrm{E}}=\frac{\omega}{4 \pi G} \frac{Q_{\mathrm{ext}} r_{b}}{\left(r_{b}^{2}+\chi^{2}\right)} .
$$

As there is no Misner string, we expect that the entropy is still given by (3.42)

$$
S_{\text {ext }}=\frac{\omega l\left(\sqrt{\kappa^{2} l^{2}+12\left(Q^{2}+P^{2}\right)}-\kappa l\right)}{24 G}
$$

which is the Bekenstein-Hawking entropy.

For $\kappa=1$ the situation is more complicated. If the Hawking temperature (3.54) is identified with the time periodicity $8 \pi \chi$ needed to eliminate the Misner string, then the zero temperature limit is approached by taking $\chi \rightarrow \infty$, which would lead us to conclude there is no zero temperature limit for the TB-AdS solution. The alternative is to abandon this identification in the extremal case since the Misner string does not appear in the nearhorizon limit and imposing time periodicity cannot be done due to the long throat of $A d S_{2}$; the Bekenstein-Hawking entropy formula is then restored even for $\kappa=1$ and we find the above thermodynamic relations hold for $\kappa=1$ as well.

Continuing with this latter assumption, we can observe new "finite temperature-like" behaviour as follows. Inspired by the idea in [23], let us identify the electric potential as a "temperature-like" quantity in this extremal limit $T_{\mathrm{H}} \rightarrow 0$. Solving (4.15) for $Q_{\text {ext }}$ and inserting this into (4.11) yields

$$
r_{b \pm}=\sqrt{\frac{\chi^{2}}{2}-\frac{\kappa l^{2}}{6}+\frac{8 \pi^{2} G^{2} l^{2}}{3 \Phi_{E}^{2} \omega^{2}} \mp \frac{\pi}{3 \omega} \sqrt{\left(\frac{\omega^{2}}{4 \pi^{2}}\left(\kappa l^{2}-3 \chi^{2}\right)-\frac{4 G^{2} l^{2}}{\Phi_{E}^{2}}\right)^{2}-\frac{12 G^{2} l^{2} \chi^{2} \omega^{2}}{\pi^{2} \Phi_{E}^{2}}}}
$$



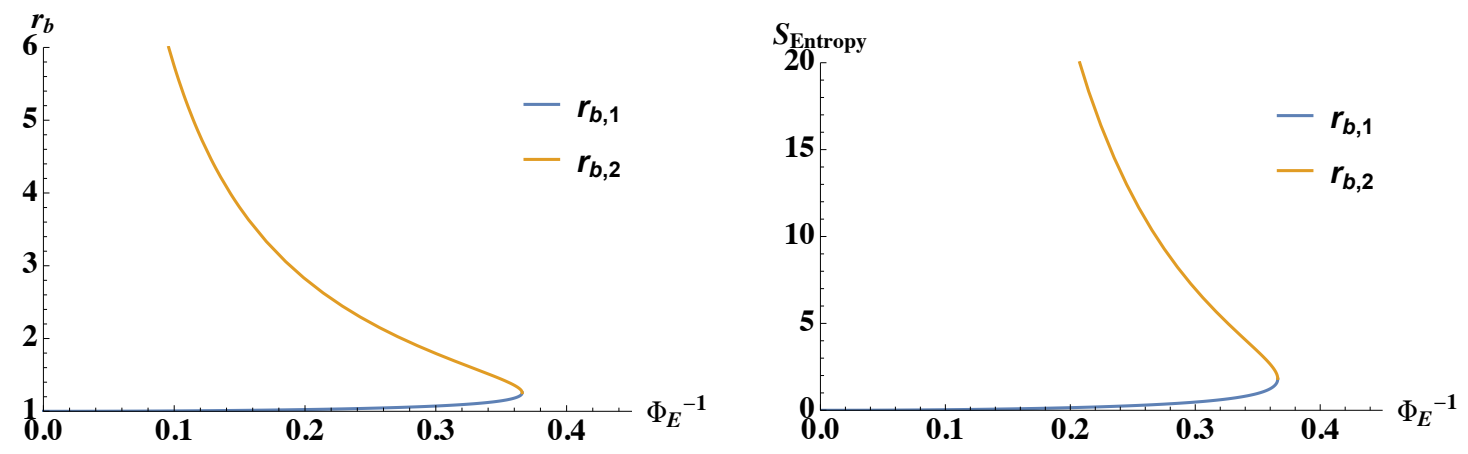

Figure 7. Left: TB-AdS radius vs. $\Phi_{E}{ }^{-1}$. Right: entropy vs. $\Phi_{E}^{-1}$ for the extremal TB-AdS for a fixed value of $\chi$ and $\kappa=1$ with $G=l=1$.

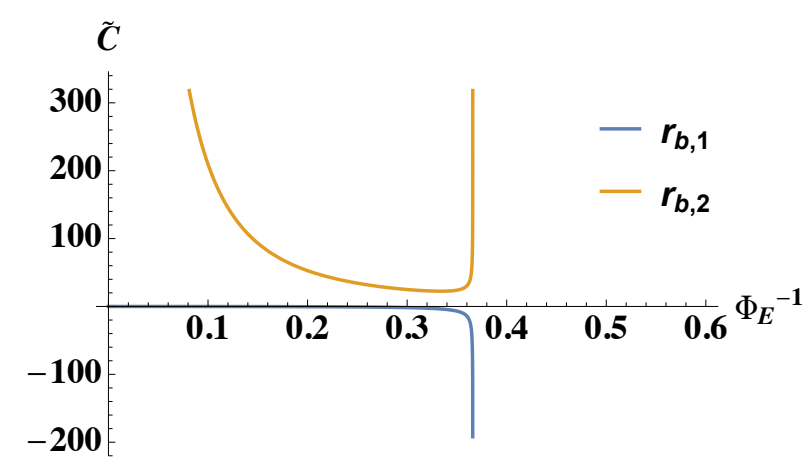

Figure 8. "Heat capacity-like" quantity vs. $\Phi_{E}^{-1}$ for the extremal TB-AdS with a fixed $\chi$ and $\kappa=1$.

as the physically acceptable values of $r_{b}$ in terms of $\Phi_{E}^{-1}$ and the other parameters. We plot $r_{b \pm}$ as a function of $\Phi_{E}^{-1}$ in figure 7 where $r_{b, 1}=r_{b-}$ and $r_{b, 2}=r_{b+}$. The resemblance to the uncharged TB-AdS behaviour displayed in figure 1 and figure 2 is clear, with the notable exception that the $x$-axis is the inverse of an electric potential and not of temperature.

We can calculate a "heat capacity-like" quantity

$$
C=\left.T \frac{\partial S}{\partial T}\right|_{Q, P} \quad \rightarrow \quad \tilde{C}=\Phi_{E} \frac{\partial S}{\partial \Phi_{E}}
$$

replacing temperature with the inverse of the electric potential. We obtain

$$
\tilde{C}=\frac{\omega r_{b}^{2}\left(r_{b}^{2}-\chi^{2}\right)\left(3 r_{b}^{2}+\kappa l^{2}-3 \chi^{2}\right)}{2 G\left(-6 \chi^{2} r_{b}^{2}+3 r_{b}^{4}-\kappa l^{2} \chi^{2}+3 \chi^{4}\right)} .
$$

and display in figure 8 the behaviour of $\tilde{C}$ as a function of $\Phi_{E}^{-1}$. We observe that the behaviour is similar to that of the uncharged TB-AdS case.

\section{Conclusion}

We have investigated thermodynamics for dyonic Euclidean Taub-NUT-AdS spacetime, extending the previous studies [19-21]. 
After reviewing the thermodynamics of the uncharged TN/TB-AdS black holes, we investigated their dyonic charged counterparts. For the charged TN-AdS, the condition $P=i Q$ is always necessary and so there is no distinction between electric and magnetic charges. For the dyonic TB-AdS, we found that the regularity condition (3.30) again made the magnetic charge dependent on the electric charge. This condition was necessary in order that the first law of thermodynamics hold. In this case, both the charged TN/TBAdS satisfy

$$
d E=T d S+\Phi_{E} d Q, \quad F=E-T S-\Phi_{E} Q
$$

and where the entropy $S$ is the Bekenstein-Hawking entropy for TB-AdS provided $\kappa \neq 1$; in this case both $r_{b}$ and $Q$ are independent parameters. For $\kappa=1$ the entropy $S$ does not follow the Bekenstein-Hawking formula due to the additional contribution of the Misner string, and because of requiring the time periodicity the phase space is reduced again. Thus the variation in the first law is made by only one variable such as $Q$. Furthermore, we explored the thermodynamic behaviours of entropy and heat capacity before imposing the regularity condition for $\kappa=1$ case. There is a maximum NUT charge, and small/large TB-AdS phases, whose radii increase/decrease as the NUT charge increases, whose heat capacity is negative/positive respectively. These characteristics appear either when the magnetic parameter is turned off and the electric charge is smaller than $0.13(l=1$ and $G=1$ ) or when the magnetic parameter becomes larger than twice the electric charge. For other parameter ranges, the behaviour is like that of the large TB-AdS.

Under this configuration we studied the zero temperature limit of TB-AdS. We showed that the near horizon geometry becomes $A d S_{2} \times \mathcal{M}_{\kappa}$, where $\mathcal{M}_{\kappa}$ is a two dimensional manifold depending on $\kappa$. This indicates that the Misner string disappears at the horizon in this limit. Retaining the standard $1 / 8 \pi \chi$ time periodicity employed at nonzero temperature implies that $\chi \rightarrow \infty$ in the zero temperature limit, in contradiction with (4.8) which would force $T \rightarrow \infty$. This contradiction indicates that there might not exist zero temperature limit in this case. However as the near horizon geometry of the extremal case is $A d S_{2} \times \mathcal{M}_{\kappa}$ (with no Misner string at the horizon), we note that time periodicity cannot be imposed on $A d S_{2}$ in this limit, suggesting we can relax this condition. So doing, we find that we can make sense of the thermodynamics in the zero temperature limit and speculate the Bekenstin-Hawking entropy formula holds even for $\kappa=1$ case. Furthermore, we showed that inverse of the electric potential can be regarded as a "temperature-like" quantity [23], with the zero-temperature TB-AdS having entropy and a "heat capacity-like" quantity exhibiting similar behaviour to the heat capacity of the uncharged TB-AdS solution at finite temperature. The full implications of abandoning time periodicity in the extremal case for $\kappa=1$ remain to be understood.

Since Taub-NUT-AdS is asymptotically locally AdS spacetime, it has drawn attention along with asymptotically AdS spacetime in AdS/CFT perspective [13, 14, 19]. The dual field theory of TN/TB-AdS space is known to be a conformal theory on a squashed sphere. It would be interesting if we find that entropy calculated at the boundary field theory recovers the gravity results under both the regularity conditions of a gauge field. 


\section{A Thermodynamics of the Dyonic RN black hole}

Here we summarize thermodynamics of dyonic RN black hole [29] and show that $\Phi_{\mathrm{E}}^{(1)}$ and $\Phi_{\mathrm{E}}^{(2)}$ yield equivalent results. The dyonic RN black hole metric has the form

$$
d s^{2}=-f(r) d t^{2}+\frac{1}{f(r)} d r^{2}+d r^{2}\left(d \theta^{2}+\sin (\theta)^{2} d \phi^{2}\right)
$$

with

$$
f(r)=1-\frac{2 M}{r}+\frac{q^{2}+p^{2}}{4 r^{2}} .
$$

The gauge field and its corresponding field strength become

$$
A=\frac{1}{\kappa}\left(\frac{q}{r}-\frac{q}{r_{h}}\right) d t-\frac{p}{\kappa} \cos \theta d \phi, \quad F=\frac{1}{\kappa}\left(\frac{q}{r^{2}} d t \wedge d r+p \Omega_{2}\right)
$$

where we fixed the gauge for $A$ to be regular at the horizon and then its norm is written as

$$
A^{2}\left(r_{h}\right)=\frac{1}{\kappa^{2}} \frac{p^{2}}{r_{h}^{2}} \cot ^{2}(\theta)
$$

The divergence that occurs at $\theta=0$ and $\pi$ in $A^{2}$ can be removed by defining the gauge field differently in the coordinate patches covering $\theta=0$ and $\pi$ so that it is regular on both. The gauge fields can be matched up to a gauge transformation where the patches overlap. All thermodynamic quantities can be calculated in a quasilocal frame [29], with the limit $R \rightarrow \infty$ taken at the end. The free energy from the semi classical approximation is then

$$
I_{\text {ren }}=\beta F=-\frac{4 \pi^{2} r_{h}\left(4 M r_{h}+p^{2}-q^{2}\right)}{\kappa^{2}\left(M-r_{h}\right)}
$$

which is equivalent to

$$
F=E-T S-\Phi_{\mathrm{E}} Q_{E}
$$

and where the various thermodynamic quantities are

$$
\begin{aligned}
& E=\frac{16 \pi}{\kappa^{2}} M, \quad T=\frac{4 M r_{h}-q^{2}-p^{2}}{8 \pi r_{h}^{3}}, \quad S=\frac{16 \pi^{2} r_{h}^{2}}{\kappa^{2}}, \\
& \Phi_{\mathrm{E}}=\frac{1}{\kappa} \frac{q}{r_{h}}, \quad \Phi_{\mathrm{M}}=\frac{1}{\kappa} \frac{p}{r_{h}}, \quad Q_{E}=\frac{4 \pi}{\kappa} q, \quad Q_{M}=\frac{4 \pi}{\kappa} p
\end{aligned}
$$

and satisfy the first law of thermodynamics

$$
d E=T d S+\Phi_{E} d Q_{E}+\Phi_{M} d Q_{M}
$$

where $Q_{E}$ and $Q_{M}$ are independent (i.e. not imposing the regularity condition on $A$ ). Here $\Phi_{\mathrm{E}}$ is obtained by $\Phi_{\mathrm{E}}^{(2)}=A_{t}\left(r_{h}\right)-A_{t}(\infty)$.

Now we calculate $\Phi_{\mathrm{E}}$ using $\Phi_{\mathrm{E}}^{(1)}=\left.\frac{\partial E}{\partial Q}\right|_{r_{h}, p}$ as in (3.31). Taking the variation of $I_{\text {ren }}$ with respect to $\beta$ first and then $Q_{E}$, we obtain

$$
\begin{aligned}
\frac{\partial I_{\mathrm{ren}}}{\partial \beta} & =E-\Phi_{\mathrm{E}}^{(1)} Q_{E}, \\
\frac{\partial^{2} I_{\mathrm{ren}}}{\partial Q_{E} \partial \beta} & =\frac{\partial E}{\partial Q_{E}}-\frac{\partial \Phi_{\mathrm{E}}^{(1)}}{\partial Q_{E}} Q_{E}-\Phi_{\mathrm{E}}^{(1)}=-\frac{\partial \Phi_{\mathrm{E}}^{(1)}}{\partial Q_{E}} Q_{E}
\end{aligned}
$$


where the first law is applied in the second line. This final expression will give us an electric potential as follows

$$
\begin{aligned}
\frac{\partial I_{\mathrm{ren}}}{\partial \beta} & =\frac{\partial M}{\partial \beta} \frac{\partial I_{\mathrm{ren}}}{\partial M}=\frac{\left(r_{h}-M\right)^{2}}{2 \pi r_{h}^{2}} \frac{\partial I_{\mathrm{ren}}}{\partial M}=\frac{2 \pi\left(4 r_{h}^{2}+p^{2}-q^{2}\right)}{\kappa^{2} r_{h}}, \\
\frac{\partial^{2} I_{\mathrm{ren}}}{\partial Q_{E} \partial \beta} & =-\frac{1}{\kappa} \frac{q}{r_{h}}=-\frac{\partial \Phi_{\mathrm{E}}^{(1)}}{\partial q} q, \\
\Phi_{\mathrm{E}}^{(1)} & =\frac{1}{\kappa} \frac{q}{r_{h}}
\end{aligned}
$$

where the last expression yields the same value of $\Phi_{\mathrm{E}}^{(2)}$ in (A.8).

\section{B Electric potential for dyonic Taub-NUT-AdS}

We now apply the same logic in appendix A to calculate the electric potential for the dyonic Taub-NUT-AdS solution. Firstly let us start with the same expression for free energy in (A.6), which is equivalent to the renormalized action value with $\beta$, then we finally obtain

$$
\frac{\partial^{2} I_{\mathrm{ren}}}{\partial Q \partial \beta}=T \frac{\partial S}{\partial Q}-\frac{\partial \Phi_{\mathrm{E}}}{\partial Q} Q=-\frac{1}{2 G} \frac{\left(Q\left(r_{b}^{2}+\chi^{2}\right)+2 i P r_{b} \chi\right) r_{b}}{\left(r_{b}^{2}-\chi^{2}\right)^{2}}
$$

where the last expression have $Q$ as a factor. This indicates that we need to start with free energy or a renormalized action having both a magnetic potential and magnetic charge

$$
I_{\text {ren }}=\beta F=\beta\left(E-T S-\Phi_{\mathrm{E}} Q-\Phi_{\mathrm{M}} P\right)
$$

from which the energy is calculated via

$$
\partial_{\beta} I_{\mathrm{ren}}=E-\Phi_{\mathrm{E}} Q-\Phi_{\mathrm{M}} P
$$

as stated in (3.35). We then calculate the electric and magnetic potentials via the definition of conjugate variables for charges in (3.31)

$$
\Phi_{E}^{(1)}=\left.\frac{\partial E}{\partial Q}\right|_{r_{b}, P}-\left.T \frac{\partial S}{\partial Q}\right|_{r_{b}, P}, \quad \Phi_{M}^{(1)}=\left.\frac{\partial E}{\partial P}\right|_{r_{b}, Q}-\left.T \frac{\partial S}{\partial P}\right|_{r_{b}, Q} .
$$

Plugging energy in (3.35) into (3.31) and making a variation with respect to $Q$ and $P$ separately, we obtain

$$
\begin{aligned}
& \partial_{Q} E=\frac{\partial^{2} I_{r e n}}{\partial Q \partial \beta}+\frac{\partial \Phi_{\mathrm{E}}^{(1)}}{\partial Q} Q+\Phi_{\mathrm{E}}^{(1)}+\frac{\partial \Phi_{\mathrm{M}}^{(1)}}{\partial Q} P, \\
& \partial_{P} E=\frac{\partial^{2} I_{r e n}}{\partial P \partial \beta}+\frac{\partial \Phi_{\mathrm{E}}^{(1)}}{\partial P} Q+\Phi_{\mathrm{M}}^{(1)}+\frac{\partial \Phi_{\mathrm{M}}^{(1)}}{\partial P} P .
\end{aligned}
$$

Since the first law is written

$$
\begin{aligned}
& d_{Q} E=T d_{Q} S+\Phi_{\mathrm{E}}^{(1)} d_{Q} Q+\Phi_{\mathrm{M}}^{(1)} d_{Q} P=T d_{Q} S+\Phi_{\mathrm{E}}^{(1)}, \\
& d_{P} E=T d_{P} S+\Phi_{\mathrm{E}}^{(1)} d_{P} Q+\Phi_{\mathrm{M}}^{(1)} d_{P} P=T d_{P} S+\Phi_{\mathrm{M}}^{(1)},
\end{aligned}
$$


Eqs. (B.3) and (B.4) can be changed to

$$
\begin{aligned}
& T d_{Q} S=\frac{\partial^{2} I_{r e n}}{\partial Q \partial \beta}+\frac{\partial \Phi_{\mathrm{E}}^{(1)}}{\partial Q} Q+\frac{\partial \Phi_{\mathrm{M}}^{(1)}}{\partial Q} P \\
& T d_{P} S=\frac{\partial^{2} I_{r e n}}{\partial P \partial \beta}+\frac{\partial \Phi_{\mathrm{E}}^{(1)}}{\partial P} Q+\frac{\partial \Phi_{\mathrm{M}}^{(1)}}{\partial P} P .
\end{aligned}
$$

Since we know $T, S$, and $I_{r e n}$, we can compute $\Phi_{\mathrm{E}}^{(1)}$ and $\Phi_{\mathrm{M}}^{(1)}$ by solving two equations (B.7) and (B.8).

\section{Solution for $r_{b}$ in terms of other parameters}

From (3.7), we set $f\left(r_{b}\right)=0$, obtaining

$$
r_{b, 1 \mp}=1-\sqrt{t 2} \chi \mp \sqrt{t 1}, \quad r_{b, 2 \mp}=1+\sqrt{t 2} \chi \mp \sqrt{t 1},
$$

where

$$
\begin{aligned}
& t 1=\frac{48 \chi^{2}-1}{\sqrt{t 2} \chi^{3}}+\frac{t 3}{(\sqrt{t 4}+\sqrt{t 5})^{1 / 3}}+\frac{4(\sqrt{t 4}+\sqrt{t 5})^{1 / 3}}{\chi}+192 \chi^{2}+\frac{1}{\chi^{2}}-32, \\
& t 2=-\frac{2 t 3}{(\sqrt{4}+\sqrt{5})^{1 / 3}}-\frac{8(\sqrt{4}+\sqrt{5})^{1 / 3}}{\chi}-96 \chi^{2}+48\left(6 \chi^{2}-1\right)+\frac{1}{\chi^{2}}+16 \\
& t 3=4 \chi\left(192\left(3 g^{2}-1\right) \chi^{2}-144 Q^{2}+576 \chi^{4}+7\right), \\
& t 4=t 5^{2}-\chi^{6}\left(192\left(3 g^{2}-1\right) \chi^{2}-144 Q^{2}+576 \chi^{4}+7\right)^{3}, \\
& t 5=27\left(192 \chi^{5}-32 \chi^{3}+\chi\right)\left(Q^{2}-4 g^{2} \chi^{2}\right)-2 \chi^{3}\left(6912 \chi^{6}-3456 \chi^{4}+414 \chi^{2}-5\right)
\end{aligned}
$$

\section{Gauge field strength}

To obtain free energy, we need to calculate the gauge field strength square $F^{2}$ in the action in Euclidean space. The indefinite $r$-integrals of each nonvanishing component of $\int d r \sqrt{g} F^{2}$ are

$$
\begin{gathered}
\left.\int d r \sqrt{g} F_{\tau r} F^{\tau r}=\left[\frac{r(4 i P Q r \chi+}{2\left(r^{2}-\chi^{2}\right)^{2}}{ }^{2}\right)\left(r^{2}+\chi^{2}\right)\right) \\
\left.-\frac{\left(Q^{2}+P^{2}\right)}{4 \chi} \log \left(\frac{r-\chi}{r+\chi}\right)\right] \sqrt{Y(\theta)^{2}} \\
\int d r \sqrt{g} F_{\theta \phi} F^{\theta \phi}=\left[\frac{r(4 i P Q r \chi+}{\left.2\left(Q^{2}-\chi^{2}\right)\left(r^{2}+\chi^{2}\right)\right)}\right. \\
\left.+\frac{\left(Q^{2}+P^{2}\right)}{4 \chi} \log \left(\frac{r-\chi}{r+\chi}\right)\right] \frac{\lambda^{\prime}(\theta)^{2}}{\sqrt{Y(\theta)^{2}}}
\end{gathered}
$$

where the range of $r$ should be taken into account in calculation of the renormalized action. 


\section{Acknowledgments}

MP would like to thank Dongsu Bak for very useful and helpful discussion and Niayesh Afshordi for the hospitality at the Perimeter Institute where part of this work was done. MP was supported by a KIAS Individual Grant (PG062001) at Korea Institute for Advanced Study and by Basic Science Research Program through the National Research Foundation of Korea funded by the Ministry of Education (NRF-2016R1D1A1B03933399). LPZ is supported in part by the U.S. Department of Energy under grant DE-SC0007859.

Open Access. This article is distributed under the terms of the Creative Commons Attribution License (CC-BY 4.0), which permits any use, distribution and reproduction in any medium, provided the original author(s) and source are credited.

\section{References}

[1] A.H. Taub, Empty space-times admitting a three parameter group of motions, Annals Math. 53 (1951) 472 [INSPIRE].

[2] E. Newman, L. Tamburino and T. Unti, Empty space generalization of the Schwarzschild metric, J. Math. Phys. 4 (1963) 915 [INSPIRE].

[3] D.R. Brill, Electromagnetic fields in a homogeneous, nonisotropic universe, Phys. Rev. 133 (1964) B845.

[4] J.F. Plebański, A class of solutions of Einstein-Maxwell equations with the cosmological constant, Symp. Int. Astron. Union 64 (1974) 188.

[5] J.F. Plebanski and M. Demianski, Rotating, charged, and uniformly accelerating mass in general relativity, Annals Phys. 98 (1976) 98 [INSPIRE].

[6] C.W. Misner, The flatter regions of Newman, Unti and Tamburino's generalized Schwarzschild space, J. Math. Phys. 4 (1963) 924 [InSPIRE].

[7] S.W. Hawking and C.J. Hunter, Gravitational entropy and global structure, Phys. Rev. D 59 (1999) 044025 [hep-th/9808085] [INSPIRE].

[8] S.W. Hawking, C.J. Hunter and D.N. Page, Nut charge, Anti-de Sitter space and entropy, Phys. Rev. D 59 (1999) 044033 [hep-th/9809035] [INSPIRE].

[9] R.B. Mann, Misner string entropy, Phys. Rev. D 60 (1999) 104047 [hep-th/9903229] [INSPIRE].

[10] M. Taylor, Higher dimensional Taub-Bolt solutions and the entropy of noncompact manifolds, hep-th/9809041 [INSPIRE].

[11] R. Clarkson, L. Fatibene and R.B. Mann, Thermodynamics of $(d+1)$-dimensional NUT charged AdS space-times, Nucl. Phys. B 652 (2003) 348 [hep-th/0210280] [INSPIRE].

[12] N. Alonso-Alberca, P. Meessen and T. Ortín, Supersymmetry of topological Kerr-Newman-Taub-NUT-AdS space-times, Class. Quant. Grav. 17 (2000) 2783 [hep-th/0003071] [INSPIRE].

[13] D. Martelli, A. Passias and J. Sparks, The supersymmetric NUTs and bolts of holography, Nucl. Phys. B 876 (2013) 810 [arXiv:1212.4618] [INSPIRE]. 
[14] C. Toldo and B. Willett, Partition functions on $3 d$ circle bundles and their gravity duals, JHEP 05 (2018) 116 [arXiv:1712.08861] [INSPIRE].

[15] G. Clément, D. Gal'tsov and M. Guenouche, Rehabilitating space-times with NUTs, Phys. Lett. B 750 (2015) 591 [arXiv:1508.07622] [INSPIRE].

[16] R.A. Hennigar, D. Kubizňák and R.B. Mann, Thermodynamics of Lorentzian Taub-NUT spacetimes, Phys. Rev. D 100 (2019) 064055 [arXiv: 1903. 08668] [InSPIRE].

[17] A.B. Bordo, F. Gray and D. Kubižnák, Thermodynamics and phase transitions of NUTty dyons, JHEP 07 (2019) 119 [arXiv: 1904.00030] [INSPIRE].

[18] A. Ballon Bordo, F. Gray and D. Kubizňák, Thermodynamics of rotating NUTty dyons, JHEP 05 (2020) 084 [arXiv: 2003.02268] [INSPIRE].

[19] A. Chamblin, R. Emparan, C.V. Johnson and R.C. Myers, Large N phases, gravitational instantons and the nuts and bolts of AdS holography, Phys. Rev. D 59 (1999) 064010 [hep-th/9808177] [INSPIRE].

[20] C.V. Johnson, Thermodynamic volumes for AdS-Taub-NUT and AdS-Taub-Bolt, Class. Quant. Grav. 31 (2014) 235003 [arXiv:1405.5941] [INSPIRE].

[21] C.V. Johnson, The extended thermodynamic phase structure of Taub-NUT and Taub-Bolt, Class. Quant. Grav. 31 (2014) 225005 [arXiv:1406.4533] [INSPIRE].

[22] L. Ciambelli, C. Corral, J. Figueroa, G. Giribet and R. Olea, Topological terms and the misner string entropy, Phys. Rev. D 103 (2021) 024052 [arXiv:2011.11044] [INSPIRE].

[23] S. Choi, J. Kim, S. Kim and J. Nahmgoong, Comments on deconfinement in AdS/CFT, arXiv: 1811.08646 [INSPIRE].

[24] D. Kubiznak, R.B. Mann and M. Teo, Black hole chemistry: thermodynamics with Lambda, Class. Quant. Grav. 34 (2017) 063001 [arXiv:1608.06147] [INSPIRE].

[25] V. Balasubramanian and P. Kraus, A Stress tensor for Anti-de Sitter gravity, Commun. Math. Phys. 208 (1999) 413 [hep-th/9902121] [INSPIRE].

[26] R. Emparan, C.V. Johnson and R.C. Myers, Surface terms as counterterms in the AdS/CFT correspondence, Phys. Rev. D 60 (1999) 104001 [hep-th/9903238] [INSPIRE].

[27] R.B. Mann and C. Stelea, On the thermodynamics of NUT charged spaces, Phys. Rev. D 72 (2005) 084032 [hep-th/0408234] [INSPIRE].

[28] W.L. Smith and R.B. Mann, Formation of topological black holes from gravitational collapse, Phys. Rev. D 56 (1997) 4942 [gr-qc/9703007] [INSPIRE].

[29] Y. Lee, M. Richards, S. Stotyn and M. Park, Quasilocal Smarr relation for an asymptotically flat spacetime, arXiv:1809.07259 [INSPIRE]. 